SH 361

.U53

- 


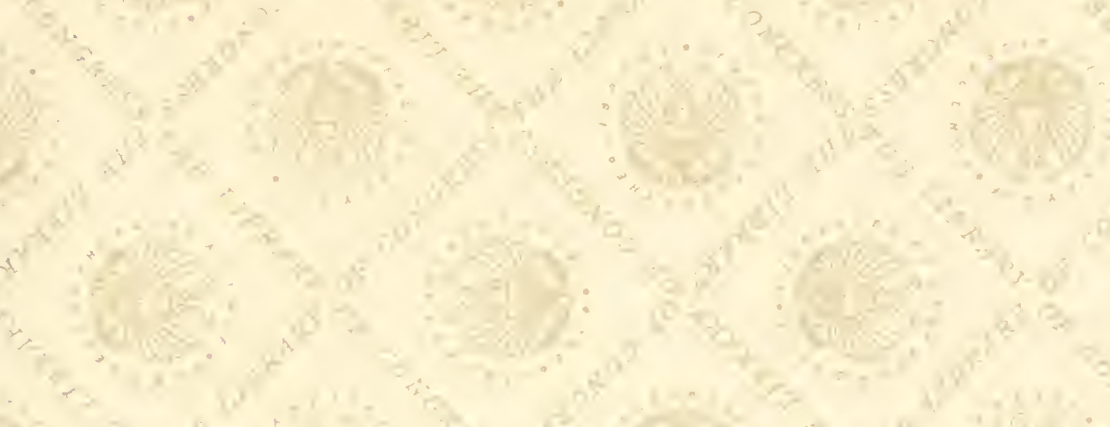

a)
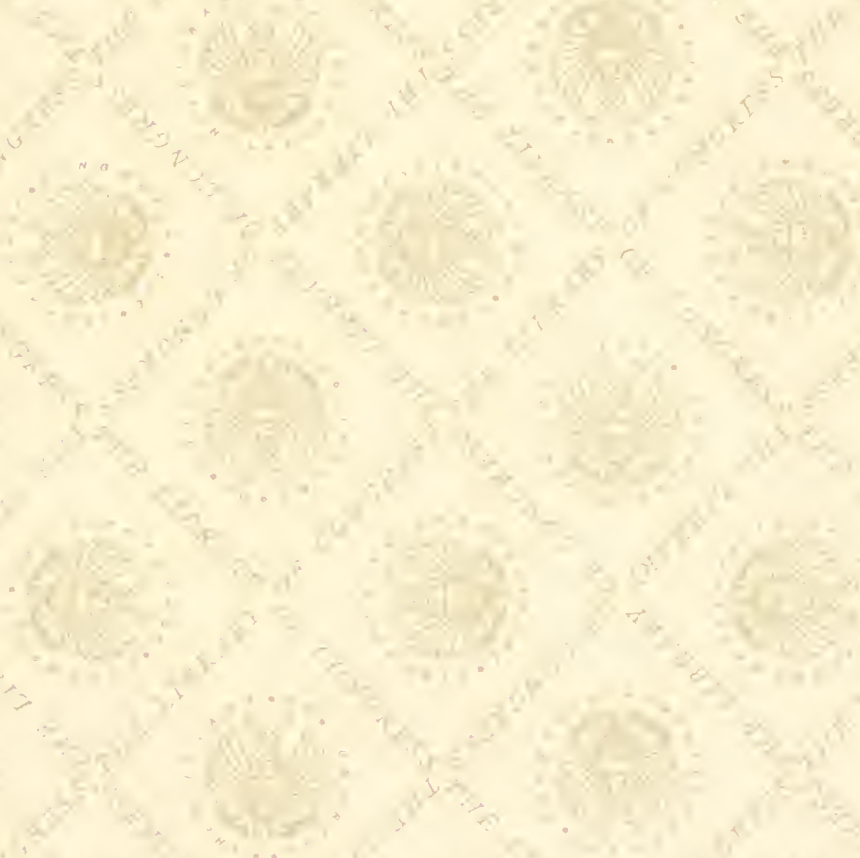

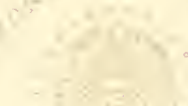






\section{STATISTICAL TABLES.}

FUR SEAL CATCH

SEASON OF 1897.

WASHINGTON :

GOVERNMENT PRINTING OFFICE.

I 898 . 

Z. S. Tress dept. Epeceial a gunts div.

\section{STATISTICAL TABLES.}

FUR SEAL CATCH

SEASON OF 1897.

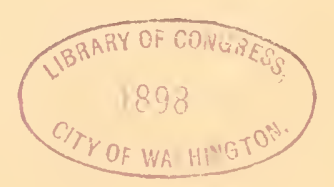

WASHINGTON :

GOVERNMENT PRINTING OFFICE.

I 898 . 


\section{-}

\section{$9+5=$ , 2h.5?}

) $6: 3 x$

Treasury Departient, Office of Secretary,

Document No. 2009.

Dirision of Special Agents. 


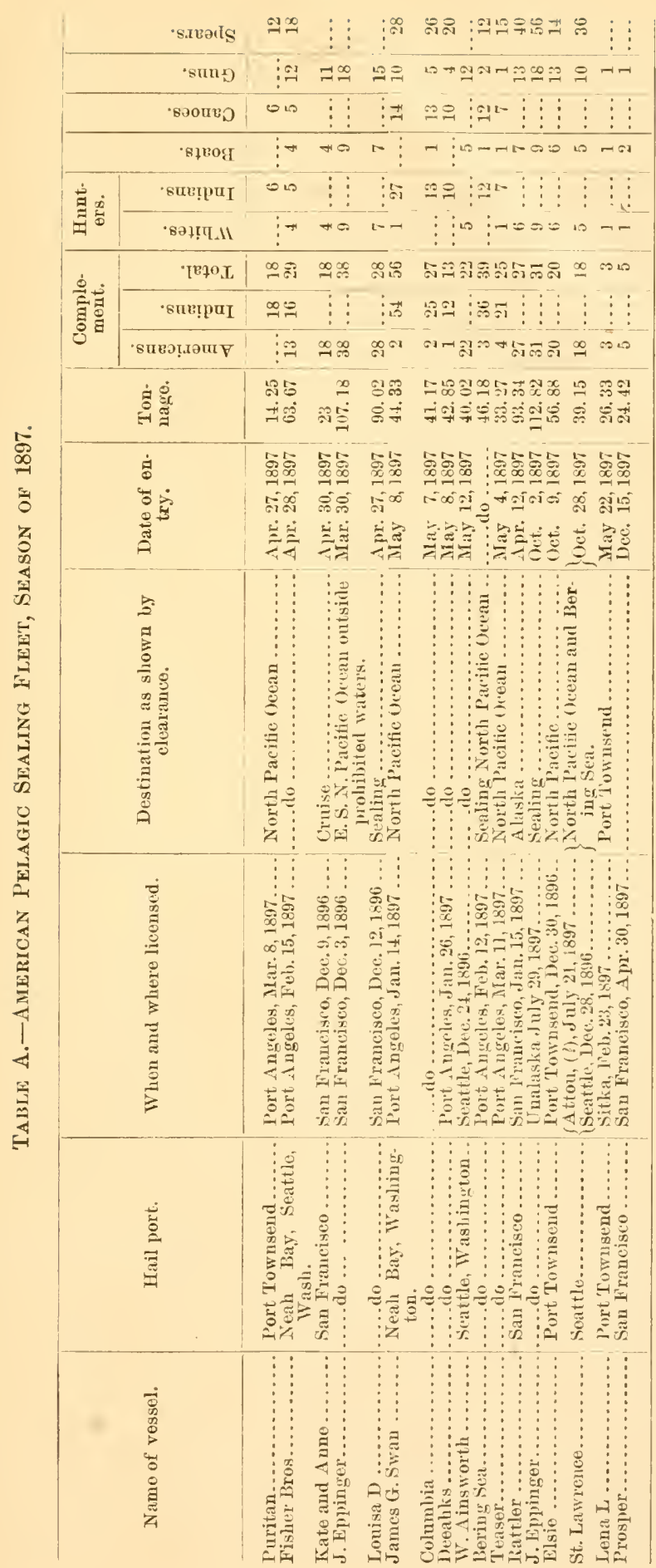




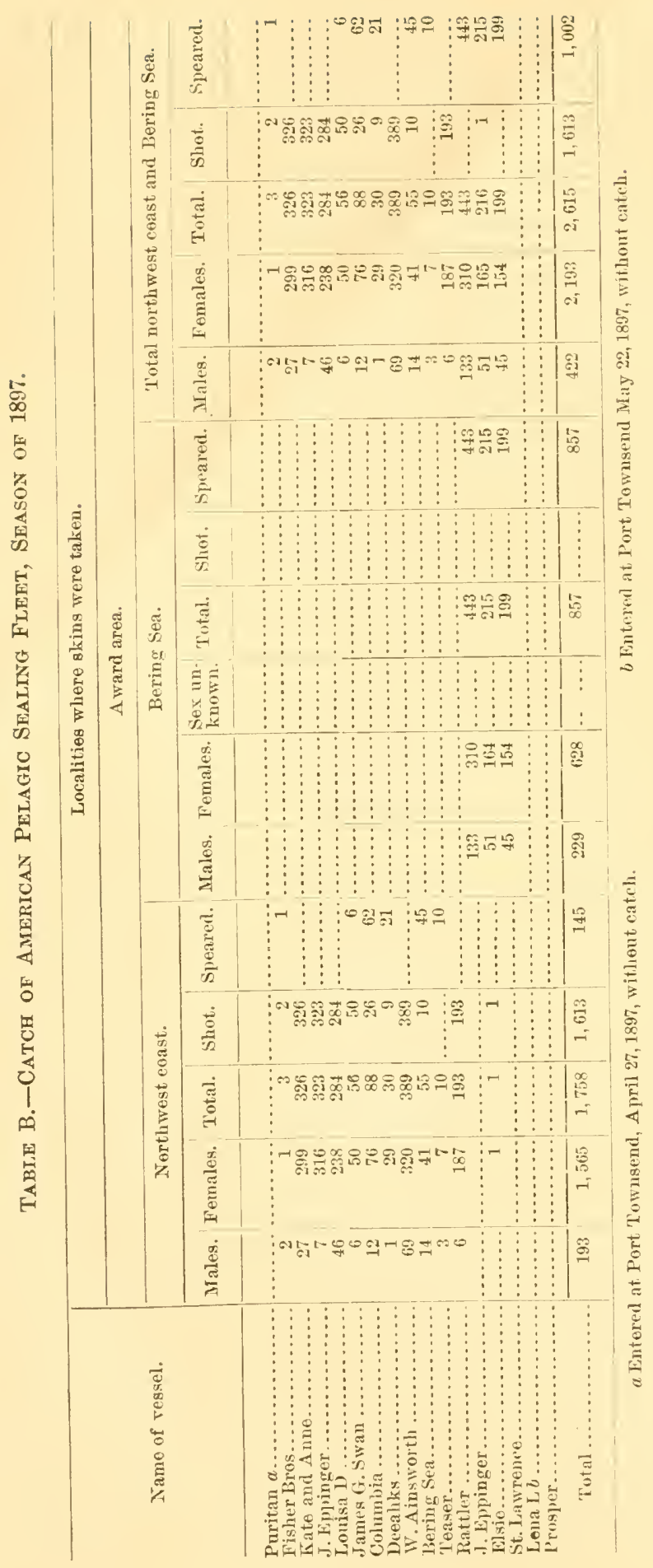


5

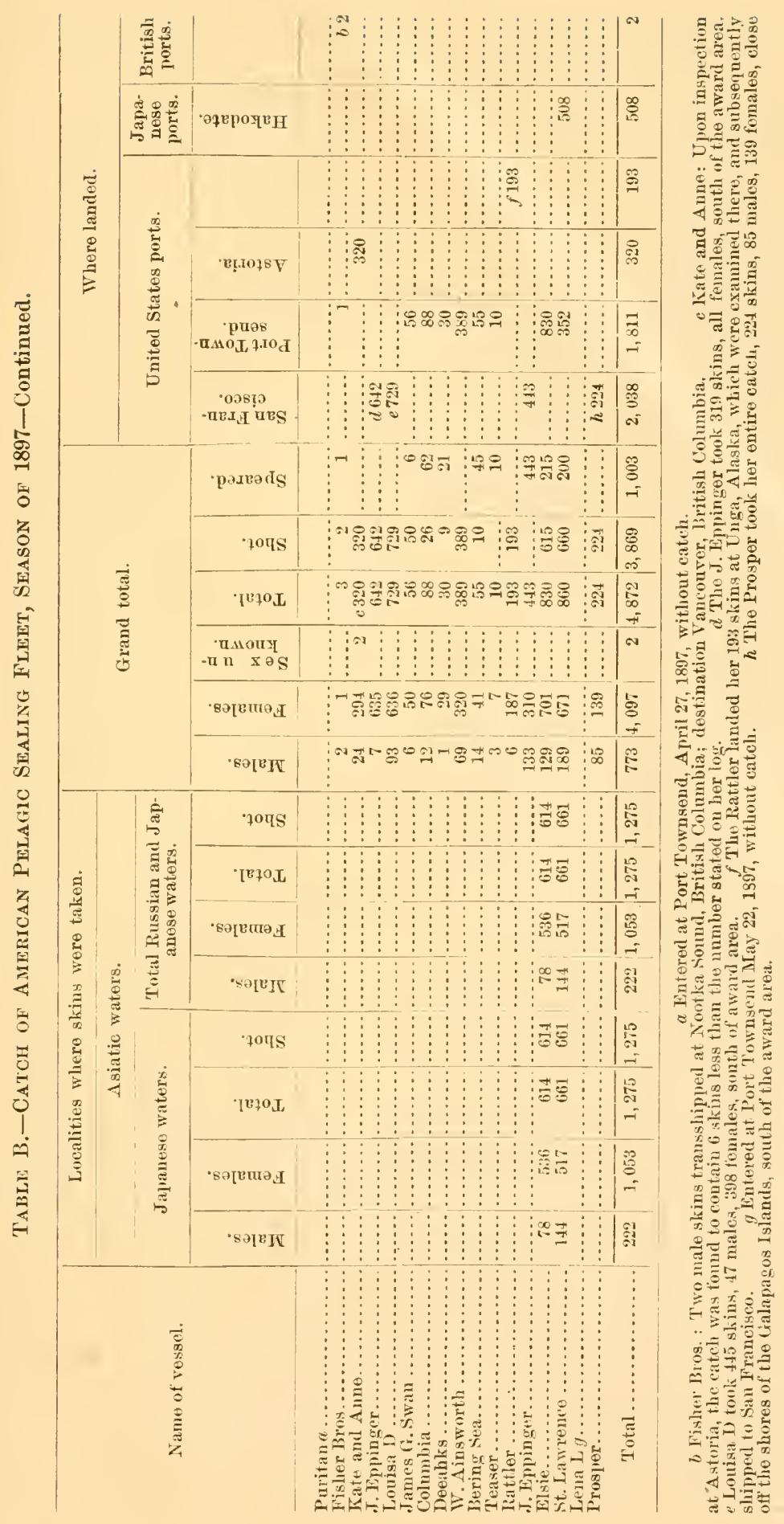




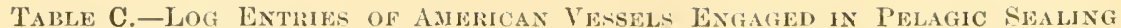

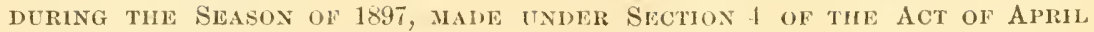
$6,1894$.

Tessel lauding her cateh at Lstoria, oreg.

\begin{tabular}{|c|c|c|c|c|c|}
\hline Name. & & Feimales. & $\begin{array}{l}\text { Sex un. } \\
\text { known. }\end{array}$ & Total. & shot. \\
\hline Kate and Anne $a . . . . .$. & 24 & 294 & 2 & 320 & 320 \\
\hline
\end{tabular}

a Upou inspection at Astoria the eatch of the Kate and Anne was found to be 6 skins less than the number stated on her log.

ABSTRACT FROM TIIE SEAL LOG BOOK OF THE SCHOONER KATE AND ANNE, ENGAGED IN FUR-SEAL FISHING TNDER SPECIAL LICENSE ISSIED AT SAN FRANCISCO, CAL.

[Cleared from San Francisco on Dec. 9, 1896; arrived from North Pacitic Ocean on A pr. 30, 1897; entered at custom-house, Astoria, (Oreg.. on Apr.30,1897.]

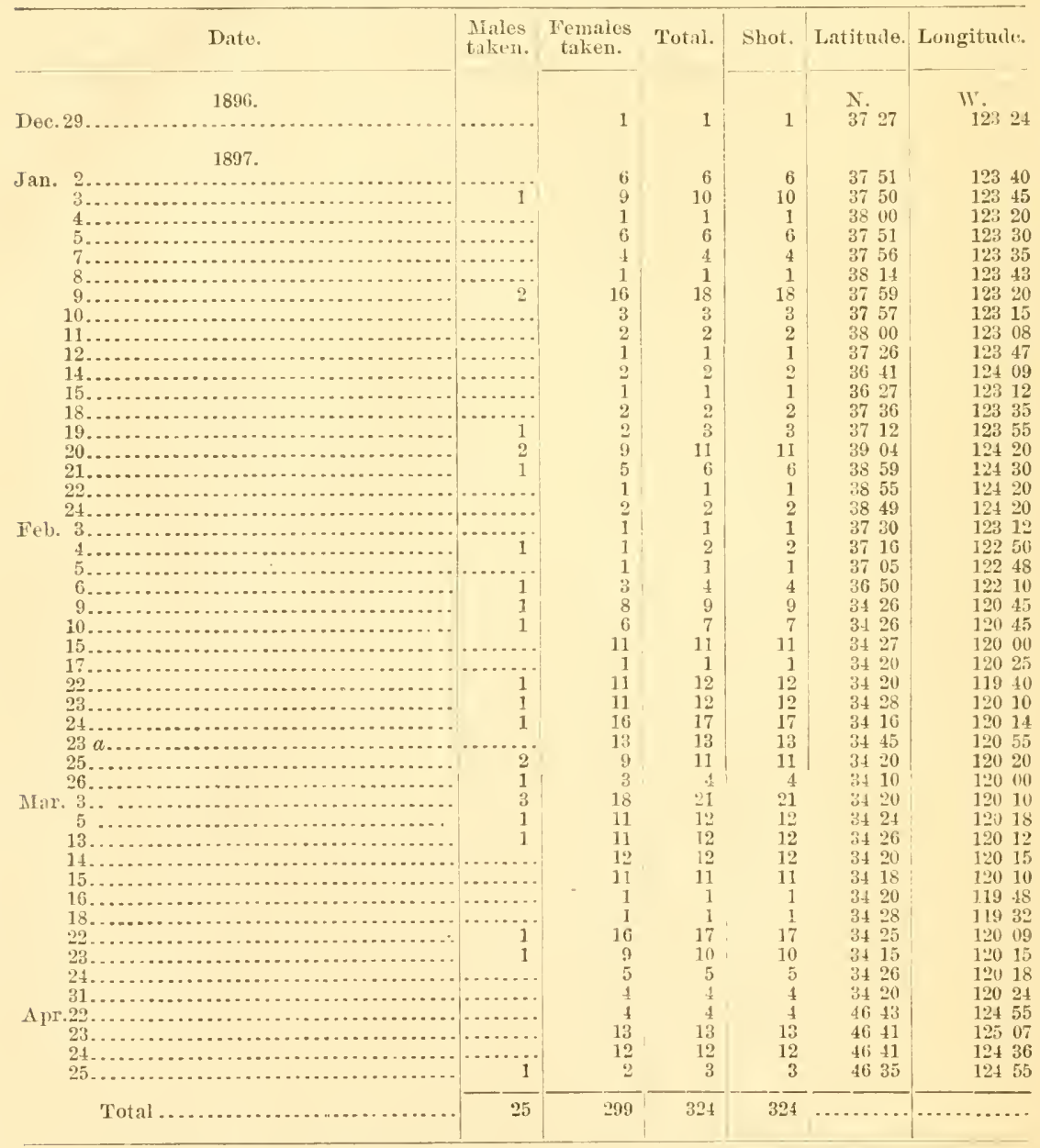

a Taken by two of the crew of the sohooner $J$. Eypingtr, whose hoat went astray and who were picknd up by Kate and Anne on February 24. 
Table C.-Log Entries of American Vessels Engaged in Pelagic Sealing DURING THE SEASON OF 1897, E'TC.-Continued.

Then personally appeared Frank Moreau, master of the within-named vessel, and made oath that the official log-book entries herein copied are a full true abstract from said book as filed by me at custom-house at Astoria, Oreg., April 29, 1897.

\section{Fraxi Mlureau.}

Subseriber and sworn to before me this 30 th day of A pril, 1897.

[SEAL.]

Chas. H. PAGE, Collector of Customs.

Tessels landing their catches at Port Townsend.

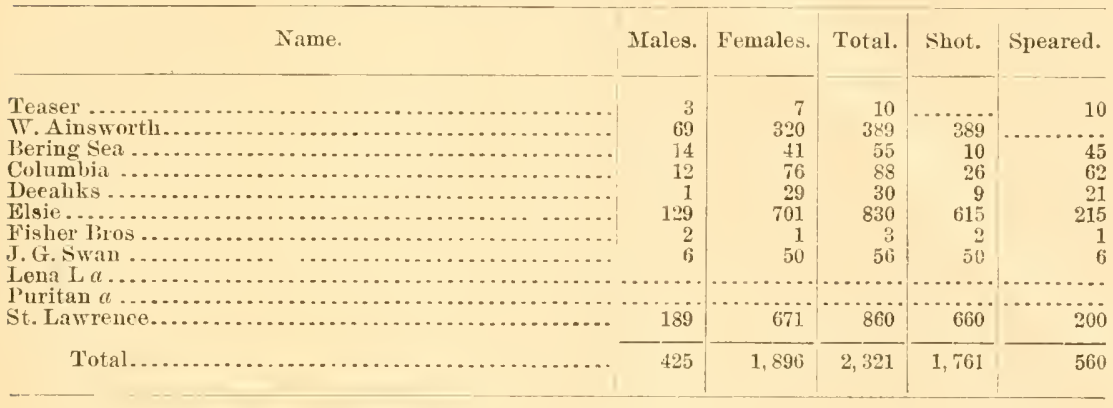

a Landed at Port Townsend without cateh.

ABSTRACT FROM 'THE SEAL LOG BOOK OF THE SCHOONER TEASER, ENGAGED IN FUR-SEAL FISHING UNDEI SPECIAL LICENSF ISSUED AT POTT ANGELES.

[Cleared from Port Angeles on Mar. 11, 1897; arrived from North Pacific Ocean on May 4, 1897; entered at custom-lionse, Seattle, Wash., ou May 5, 1897.]

\begin{tabular}{|c|c|c|c|c|c|c|}
\hline Date. & $\begin{array}{l}\text { Males } \\
\text { taken. }\end{array}$ & $\begin{array}{c}\text { Females } \\
\text { taken. }\end{array}$ & Total. & Speared. & Latitude. & Lougitude. \\
\hline 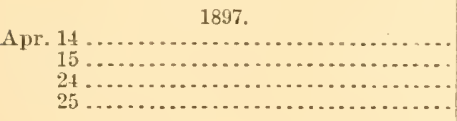 & $\begin{array}{r}1 \\
1 \\
1 \\
1\end{array}$ & $\begin{array}{r}3 \\
3 \\
1\end{array}$ & $\begin{array}{l}4 \\
1 \\
3 \\
2\end{array}$ & $\begin{array}{l}4 \\
1 \\
3 \\
2\end{array}$ & $\begin{array}{ll}\text { N. } & \\
47 & 48 \\
48 & 06 \\
47 & 55 \\
47 & 38\end{array}$ & $\begin{array}{ll}\mathbb{T} & \\
125 & 12 \\
125 & 11 \\
125 & 05 \\
125 & 48\end{array}$ \\
\hline Total ........ & 3 & 7 & 10 & 10 & $\ldots$ & $\ldots \ldots$ \\
\hline
\end{tabular}

Port Townsend, IVAsil., May 5, 1897.

Then personally appeared Mark Olsen, master of the within-named vessel, and made oath that the official log-bonk entries herein referred to, and any copies thereof herewith filed, and the answers to the forecroing questions marle by hin are true, and the whole truth, to the best of his knowledge and belief.

liefore me,

[SEAL.]

F. A. McDonali, Deputy Collector. 
Table C.-Log Extries of American Vessels Engaged in Pelagic Sealing DURing the Season of 1897, ETc.-Continued.

Port of Port Townsend, Wash., May 1\%, $189 \%$.

I certify that the within is a true copy of the return on file in the customs office, port of l'ort 'Townsend, Wash., and that the copy of the official log-book entries hereto annexed is a true copy.

\section{J. C. Sauniners, Collector Port of Port Townsend.}

ABSTRACT FROM THE SEAL LOG BOOK OF THE SCHOONER WILLARD AINSWORTH, ENGAGED IN FUR-SEAL FISHING UNDER SPECIAL LICENSE ISSUED AT SEATTLE, WASH.

[Cleared from Seattle on Dec. 24, 1896; arriced from North Pacific Ocean on May 12, 1897; entered at custom-house, Seattle, Wash., on May 12, 1897.]

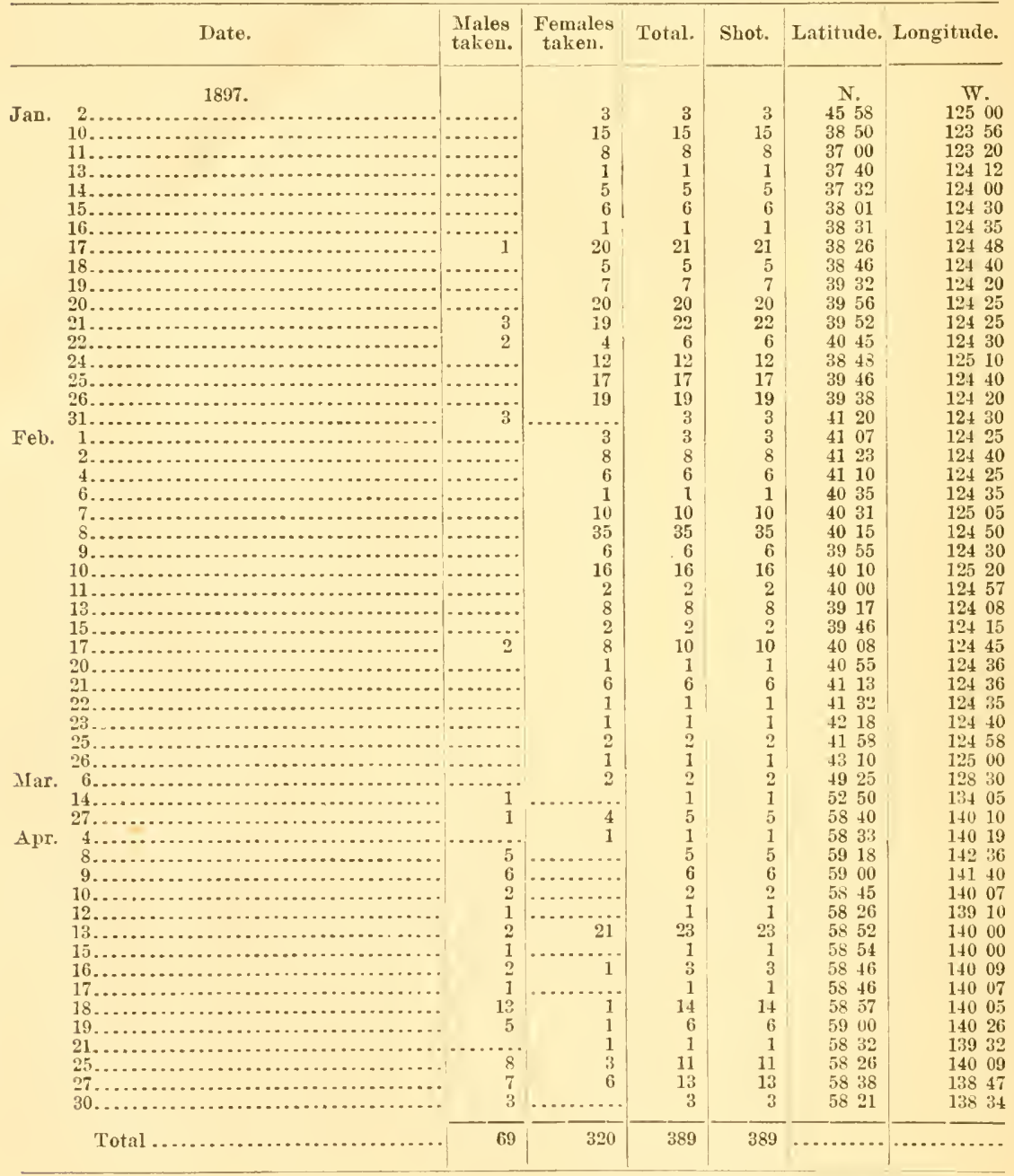

Siattle, Wash., May 1\%, 189\%.

Thon personally appeared E. E. Crockett, master of the within-namerl vessel, and mado oath that the official log-book entries herein reforred to, aud any copies thereof herewith filed, and the answers to the foregoing questions made by him are true, and the whole truth, to the best of his knowledge and belief. Before me sworn to Mav 12, 1897.

[SEAL.]

F. A. McDonald, Deputy Collector. 
'Table C.-Log Extries of American Vessels Engaged in Pelagic Sealing DURING THE SEASON OF 1897, ETC.-Continued.

Port of Port Townsexil, Wash., May 17, 1897.

I certify that the within is a true colyy of the return on file in the customs office, port of Port 'Towusend, Wash., and that the copy of the official log-bookentries hereto annexed is a true copy.

J. C. SAUNDERS,
Collector Port of Port Tounsend, ITash.

ABSTRACI FRON THE SEAL LOG BOOK OF THE SCHOONER BERING SEA. ENGAGED IN FUR-SEAL FISHING UNDER SPECIAL LICENSE ISSUED AT PORT INGELES.

[Cleared from Port Angeles on Feb.12, 1897: arrived from North Pacific Ocean on May 4, 1897; entered at custom-house, Seattle, Wash., on May 4, 1897.]

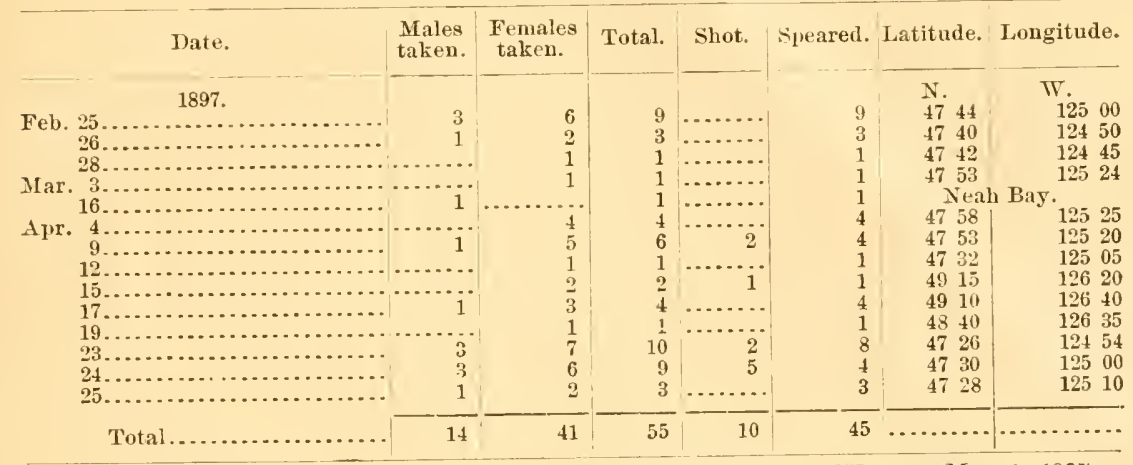

Port Townsend, Wasir., Muy 4, 1897.

Then personally appeared L. Larsen, master of the within-named vessel, and made oath that the official log-book entries herein referred to, and any copies thereof herewith filed, and the answers to the foregoing questions made by him are true, and the whole truth, to the best of his knowledge and belief.

Before me,

F. A. McDonat.o, Deputy Collector.

Filed and recorded May, 1897.

Port of Port Townsend, Wash., May 1?, 1897.

I certify that the within is a true copy of the return on file in the custons office, port of Port Townsend, Wash., and that the copy of the official log-book entries hereto annexed is a true copy.

J. C. SAUNDERS,

Collector Port of Port Tou'nsend.

ABSTRACT FlROM THE SEAL LOG BOOK OF THE SCHOONER COLCMBIA, ENGAGED IN FUR-SEAL FISIING UNDER SPECIAL LICENSF ISSUED AT PORT AXGELES.

[Cleared from Port Angeles on Jan. 14, 1897; arrived from North Pacifie Ocean on May 1, 1897; entered at custom-house. Port 'Townsend, on May 1, 1897. ]

\begin{tabular}{|c|c|c|c|c|c|c|c|c|}
\hline & Date. & $\begin{array}{l}\text { Males } \\
\text { taken. }\end{array}$ & $\begin{array}{c}\text { Females } \\
\text { taken. }\end{array}$ & Total. & shot. & speared. & Latituile. & Longitude. \\
\hline Feb & 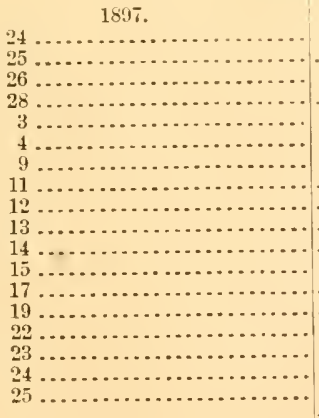 & 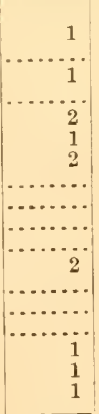 & $\begin{array}{r}5 \\
4 \\
12 \\
1 \\
2 \\
2 \\
7 \\
1 \\
1 \\
1 \\
4 \\
17 \\
4 \\
4 \\
3 \\
1 \\
7 \\
\ldots . . \\
\end{array}$ & $\begin{array}{r}6 \\
4 \\
13 \\
1 \\
4 \\
3 \\
9 \\
1 \\
1 \\
1 \\
4 \\
19 \\
4 \\
4 \\
3 \\
2 \\
8 \\
1\end{array}$ & 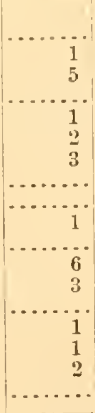 & $\begin{array}{r}6 \\
3 \\
8 \\
1 \\
3 \\
1 \\
6 \\
1 \\
1 \\
\ldots \\
4 \\
13 \\
1 \\
4 \\
2 \\
1 \\
6 \\
1 \\
\end{array}$ & $\begin{array}{ll} & N \\
48 & 00 \\
47 & 14 \\
47 & 36 \\
48 & 20 \\
47 & 47 \\
47 & 54 \\
47 & 42 \\
47 & 29 \\
47 & 35 \\
47 & 48 \\
47 & 34 \\
48 & 53 \\
49 & 15 \\
48 & 50 \\
48 & 23 \\
47 & 50 \\
47 & 43 \\
47 & 23\end{array}$ & $\begin{array}{ll}\text { II } \\
125 & 30 \\
125 & 20 \\
125 & 02 \\
125 & 15 \\
125 & 20 \\
125 & 21 \\
125 & 18 \\
125 & 30 \\
125 & 50 \\
125 & 20 \\
125 & 40 \\
126 & 20 \\
126 & 55 \\
126 & 20 \\
126 & 05 \\
125 & 03 \\
125 & 05 \\
125 & 06\end{array}$ \\
\hline & Total.......................... & 12 & 76 & 80 & & U & & \\
\hline
\end{tabular}




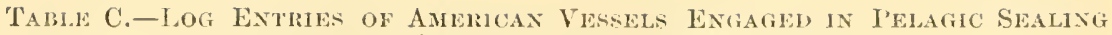
DURA THE SEASON OF 1897, ETC.-Continned.

Port Townswil, Wash., May 1, 189 .

'Then persomally appeared C. Peterson, master of the within-named ressel, and made oath that the oficial log-book entries herein referred to, and any copies thereof herewith filed, and the answers to the foregoing questions made by him are true, and the whole truth, to the best of his knowledge and belief.

Before me, Nay 1, 1897.

Filed and recorted May 1, 1897.

Cuas. Minder, Deputy Collector.

Port of l'ort Townsexd, Wail., Lay $S, 1897$.

I certify that the within is a trne copy of the retum on file in the customs office, port of Port Townsend, Wash., and that the copy of the official log-book entries hereto annexed is a true col'y.

\section{J. C. Sa UNmers.}

Collector Port of I'ort Townsend, Wash.

ABSTLACT FROM TIE SEAL LOG BOOK OF THE DEEAHKS. FNGAGED IN FTR. SEAL FISHTNG UNIER SPEC1AL LICENSE ISSUED A'T POLT ANGELES.

[Cleared from Port Angeles 0n Jan. 26, 1897; arriced from North I'acific Ocean on May 1, 1897; entered at custom-house Port Townsend on May 1, 1897.]

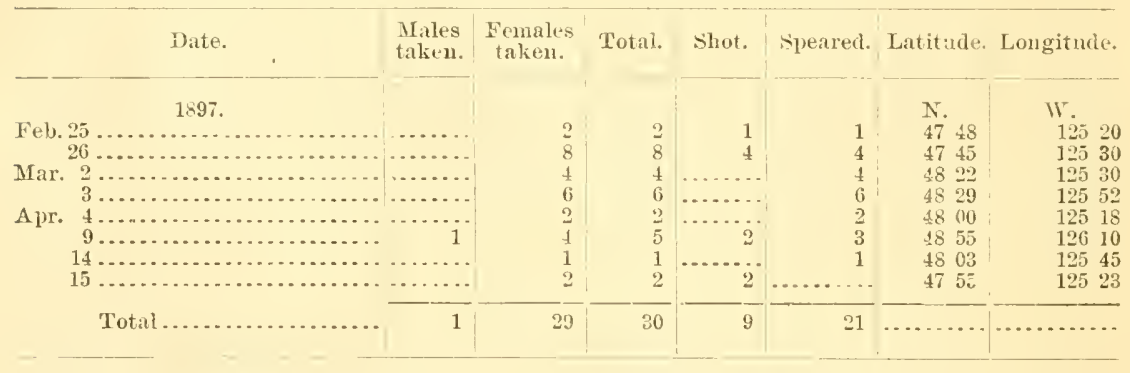

PoRT Townsend, WAsil., May 1, $189 \%$.

Then personally appeared James Claplanboo, master of the within-named vessel, and made oath that the official log-book entries berein referred to, and any copies thereof herewith filed, and the answers to the foregoing questions malle by him are true, and the whole truth, to the best of his knowlerlge and belief.

Before me swom to Mlay 1, 1897.

Filed and recorded May 1, 1897.

Chas. Miluer, Deputy Collector.

Pont of l'ort Townsenn, Wash., May $\mathcal{S}, 189 \%$.

I certify that the within is a true cony of the return on file in the rustoms office, port of l'ort Townsend, Wash., and that the copy of the official log-book entries hereto anmexed is a true copy.

J. C. SAUNDERS,

Collector l'ort of l'ort Tounsend, llash. 
Table C.-Log Extrifis of American Tessels Engaged in Pelagic sealing DEHING TIE SEASON OF 1897 , ETC.-Continued.

ABSTRACT FROM THE SEAL LOG BOOK OF THE SCHOONEL ELSIE, ENGAGED IN FUL-SEAL FISIING TNDER SPECIAL LICENSE ISSUED A'T ATTU, ALASKA.

[Cleared from Port Townsend on Lec. 30, 1896; arrived from Bering Sea on Oct. 2, 1897; entered at custom-house, Port Townsend, on Oet. 2, 1897.]

\begin{tabular}{|c|c|c|c|c|c|c|c|c|}
\hline & Date. & $\begin{array}{l}\text { Males } \\
\text { talien. }\end{array}$ & $\begin{array}{l}\text { Females } \\
\text { taken. }\end{array}$ & Total. & Sliot. & Speared. & Latitucle. & Longitur \\
\hline & 1897 & & & & & & $\mathrm{~N}$ & II \\
\hline $\begin{array}{l}\text { Feb. } \\
\text { Apr. }\end{array}$ & $1, \ldots \ldots \ldots \ldots \ldots \ldots \ldots \ldots$ & & 1 & 1 & 1 & $\cdots \ldots \ldots$, & 3732 & 12432 \\
\hline A pr. & $1, \ldots \ldots \ldots$ & $\cdots$ & 2 & 2 & 2 & .......... & 3543 & $146 \quad 34$ \\
\hline & 6 & $\begin{array}{l}4 \\
1\end{array}$ & $\begin{array}{r}63 \\
6\end{array}$ & 67 & 67 & n........ & $37+6$ & 14354 \\
\hline & 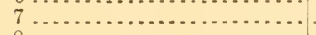 & & $\begin{array}{l}0 \\
1\end{array}$ & 1 & i & (n. & $\begin{array}{l}3759 \\
38 \quad 05\end{array}$ & $\begin{array}{l}14347 \\
14340\end{array}$ \\
\hline & $8 \ldots \ldots \ldots \ldots \ldots \ldots \ldots$ & & 9 & 9 & 9 & ......... & 3742 & $1430 \%$ \\
\hline & $13 \ldots \ldots \ldots \ldots \ldots \ldots \ldots+\ldots$ & 3 & 8 & 11 & 11 & $\ldots \ldots \ldots$ & 3839 & 14159 \\
\hline & $15 \ldots \ldots \ldots \ldots$ & 5 & 1 & 1 & 1 & $\ldots \ldots \ldots$ & 3820 & $142 \div 0$ \\
\hline & 17 18 & 5 & 11 & 16 & 16 & ........... & 3742 & 14236 \\
\hline & $\begin{array}{l}18, \ldots \\
19\end{array}$ & & 1 & 1 & 1 & $\ldots \ldots \ldots$ & 3806 & 14322 \\
\hline & $\begin{array}{l}19 \\
30 \\
30\end{array}$ & 2 & 4 & 6 & 6 & $\ldots \ldots \ldots$ & 3736 & 14340 \\
\hline & $21{ }_{2}{ }_{2}$ & 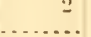 & 12 & 14 & 14 & (.......... & $37+5$ & 14253 \\
\hline & $25 \quad 15 \ldots$ & $\begin{array}{l}\cdots \\
\cdots\end{array}$ & 13 & 13 & 13 & $\ldots \ldots \ldots$ & 3726 & 14254 \\
\hline & 27 .... & $\because$ & 2 & 2 & 2 & $\ldots \ldots$ & $\begin{array}{ll}38 & 19 \\
2 & -9\end{array}$ & 14531 \\
\hline & $30 \ldots \ldots \ldots \ldots \ldots \ldots \ldots \ldots \ldots$ & $\begin{array}{l}1 \\
3\end{array}$ & $\ddot{i}$ & $1 !$ & 1 & $\cdots \cdots+\cdots$ & 3737 & 4730 \\
\hline Mas & $1, \ldots \ldots \ldots \ldots$ & 7 & 44 & 51 & 51 & (n) & $\begin{array}{ll}37 & 47 \\
37 & 32\end{array}$ & 4439 \\
\hline & $2 \ldots \ldots \ldots \ldots \ldots \ldots \ldots \ldots$ & 17 & 82 & 99 & 99 & (n) & $37 \begin{array}{l}32 \\
37 \\
37\end{array}$ & $\begin{array}{l}14355 \\
143+5\end{array}$ \\
\hline & $3 \ldots \ldots \ldots \ldots \ldots$ & - . & 1 & 1 & 1 & ......... & 3734 & $\begin{array}{ll}143 & 45 \\
144 & 00 ! 3\end{array}$ \\
\hline & $5 \ldots$ & $\cdots$ & 1 & 1 & 1 & $\ldots \ldots \ldots$ & 3728 & 14329 \\
\hline & $6 \ldots \ldots \ldots \ldots \ldots \ldots \ldots . . . \ldots \ldots$ & 7 & 18 & 25 & 25 & $\ldots \ldots . . .$. & 3733 & 14348 \\
\hline & $7 \quad \ldots+\ldots$ & 3 & 13 & 16 & 16 & ......... & 3714 & 14322 \\
\hline & $9 \quad \ldots \ldots \ldots \ldots$ & 5 & 12 & 17 & 17 & ......... & 3737 & 14401 \\
\hline & $10 \ldots \ldots \ldots \ldots \ldots \ldots$ & $\ldots$ & 2 & 2 & 2 & $\ldots \ldots \ldots$ & 3716 & 14356 \\
\hline & $11 \ldots \ldots \ldots \ldots \ldots \ldots \ldots$ & 53 & 18 & 71 & 71 & $\ldots \ldots \ldots$ & 3742 & 14358 \\
\hline & $12 \ldots \ldots \ldots \ldots \ldots \ldots \ldots \ldots$ & 35 & $1:$ & $\frac{4}{48}$ & 48 & $\ldots \ldots \ldots$ & 3728 & 11233 \\
\hline & $13, \ldots \ldots \ldots \ldots \ldots \ldots \ldots$ & 3 & 57 & 60 & 60 & $\ldots \ldots \ldots$ & อ7 11 & $1+2 \div \overline{3}$ \\
\hline & $14 \ldots \ldots \ldots \ldots \ldots$ & 2 & 6 & 8 & 8 & 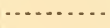 & 3731 & 14230 \\
\hline & $17 \quad \ldots \ldots$ & $\cdots$ & 1 & 1 & $\because$ & .......... & 2903 & 14636 \\
\hline & $19 \ldots \ldots \ldots \ldots \ldots \ldots$ & - & 2 & 2 & 2 & $\ldots \ldots \ldots$ & 3924 & 14748 \\
\hline & $\frac{21}{2} \ldots \ldots \ldots \ldots \ldots \ldots \ldots$ & 1 & 1 & 2 & 2 & ........... & 3940 & 14745 \\
\hline & 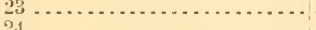 & $\because$ & 7 & 7 & 7 & . $\ldots \ldots \ldots$ & 3805 & 14548 \\
\hline & $\frac{24}{0} \ldots \ldots \ldots \ldots \ldots \ldots \ldots \ldots \ldots$ & 1 & 1 & 2 & 2 & (n........... & 3917 & 4500 \\
\hline & $25, \ldots \ldots+\ldots \ldots \ldots \ldots$ & 7 & 4 & 11 & 11 & (........ & 4044 & 14413 \\
\hline & 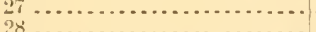 & & 1 & 1 & 1 & .......... & 4118 & $1 \frac{13}{43} 35$ \\
\hline & $\begin{array}{l}28 \\
29\end{array}$ & 3 & 1 & 4 & 4 & $\ldots \ldots \ldots$ & 4130 & 14335 \\
\hline June & (1) & 1 & $\cdots$ & 1 & 1 & $\ldots \ldots \ldots$ & $42 \quad 19$ & 14353 \\
\hline June & 3 & ] & $\cdots$ & 1 & 1 & $\ldots \ldots \ldots$ & 4410 & 14718 \\
\hline & ( & & 4 & 4 & 4 & .......... & 4418 & 14729 \\
\hline & 5 . . & 3 & $\begin{array}{l}4 \\
3\end{array}$ & 6 & 6 & $\ldots \ldots \ldots$ & $4 \frac{1}{16}$ & 14724 \\
\hline & $8 \ldots \ldots \ldots \ldots$ & $\begin{array}{l}3 \\
1\end{array}$ & 3 & 6 & 6 & $\ldots \ldots \ldots$ & 4409 & 14722 \\
\hline & $9 \ldots \ldots \ldots \ldots \ldots \ldots$ & $\begin{array}{l}1 \\
1\end{array}$ & 1 & 1 & $\frac{1}{2}$ & (2.). & 4414 & \\
\hline 1 & $10 \ldots \ldots \ldots \ldots \ldots \ldots$ & 1 & 3 & $\frac{2}{4}$ & 4 & - & 4410 & 14732 \\
\hline & $11 \ldots \ldots \ldots \ldots \ldots \ldots$ & 1 & & 1 & 1 & - & 4417 & $147 \geq 8$ \\
\hline & $25, \ldots \ldots \ldots \ldots \ldots \ldots \ldots \ldots \ldots \ldots$ & 1 & 2 & 3 & 3 & $\ldots$ & 5047 & 15815 \\
\hline Aug. & $1 \ldots \ldots \ldots \ldots \ldots \ldots \ldots$ & $\cdots$ & 1 & 1 & $\ldots \ldots$. & 1 & 5524 & 17150 \\
\hline & $2 \quad \ldots \ldots \ldots \ldots \ldots \ldots$ & 1 & 9 & 10 & $\ldots \ldots$. & 10 & 5540 & 17159 \\
\hline & $5 \ldots \ldots \ldots \ldots \ldots \ldots \ldots \ldots \ldots \ldots \ldots$ & 1 & & 1 & $\ldots \ldots \ldots$ & 1 & $56 \quad 10$ & 17248 \\
\hline & $6 \ldots \ldots \ldots \ldots \ldots$ & $\therefore$ & 11 & 11 & $\ldots \ldots \ldots$ & 11 & 5536 & 17242 \\
\hline & 7 & 2 & 4 & 6 & $\ldots \ldots \ldots$ & 6 & 5548 & 17130 \\
\hline & $11 \ldots \ldots \ldots \ldots \ldots \ldots \ldots \ldots \ldots \ldots$ & 4 & 15 & 19 & $\ldots \ldots \ldots$ & 14 & 5757 & 17334 \\
\hline & $12 \quad \ldots+\ldots$, & 1 & 4 & 5 & $\ldots \ldots$. & 5 & 5758 & 17349 \\
\hline & $16 \ldots \ldots \ldots \ldots \ldots \ldots$ & 3 & 3 & 6 & $\ldots \ldots$ & 6 & 5809 & 17313 \\
\hline & 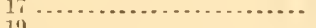 & 1 & 1 & 1 & ....... & 1 & 5804 & 17329 \\
\hline & (n. & 1 & 1 & 12 & - $\ldots \ldots$ & 12 & 5867 & 17318 \\
\hline & 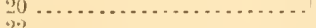 & 2 & 2 & 23 & . . . . . & $2: 3$ & 5806 & 17302 \\
\hline & 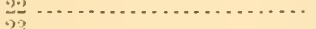 & $\begin{array}{l}5 \\
5\end{array}$ & 12 & 17 & $\ldots \ldots \ldots$ & 17 & $58 \quad 08$ & 17300 \\
\hline & 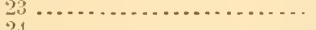 & 2 & 5 & 7 & $\ldots \ldots \ldots$ & 7 & 5831 & 17301 \\
\hline & $24, \ldots \ldots \ldots \ldots \ldots \ldots \ldots \ldots \ldots \ldots \ldots$ & $\because$ & 9 & 9 & - . . . & 9 & 5756 & 17259 \\
\hline & (n) & 3 & 23 & 36 & . . . . . & 26 & $58 \quad 01$ & 17335 \\
\hline & $26 \ldots \ldots \ldots \ldots \ldots$ & $\cdots$ & 8 & 8 & $\ldots \ldots \ldots$ & 8 & $58 \quad 03$ & 17330 \\
\hline & $28 \ldots \ldots \ldots \ldots \ldots$ & 6 & 12 & 18 & - . . - & 18 & 5.343 & $172 \quad 32$ \\
\hline & 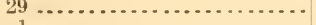 & & 1 & 1 & $\ldots \ldots$ & 1 & 5538 & 17236 \\
\hline sept. & $1, \ldots \ldots \ldots$ & 4 & 2 & 6 & $\ldots \ldots$ & 6 & 5540 & 17106 \\
\hline & $2 \ldots \ldots \ldots \ldots \ldots \ldots \ldots$ & 1 & 1 & 3 & $\cdots$. & 2 & 5520 & 17140 \\
\hline & $3 \ldots \ldots \ldots \ldots, \ldots$ & 9 & 8 & 17 & ........ & 17 & 5436 & $169 \div 7$ \\
\hline & (., & 1 & & 1 & $\ldots \ldots \ldots$ & 1 & $\begin{array}{ll}55 & 03\end{array}$ & 16747 \\
\hline & $\ldots \ldots \ldots \ldots \ldots$ & 5 & 3 & 8 & $\ldots \ldots \ldots$ & 8 & 5506 & 16706 \\
\hline & Total.................. & 229 & 601 & 830 & 615 & 10 & & \\
\hline
\end{tabular}


Table C.-Log Extries of Americin Vessels Exgagen in l'elagic Sealing DURING TIE SEASON OF 1897, ETC.-Continuel.

ABSTRACT FROM THE SEAL LOG BOOK OF THE FISCIIER BROS., ENGAGED IN FURSEAL FISIING TNIER SPECIAI, LICENSE ISSTED AT SEATTLE, WASH.

[Cleared from Seatle on Feb.15, 1897, arriced from North Pacific Ocean on Apr. 28, 1897, entered at eustom-house, Seattle, Wish., on A pr. 28, 1897.]

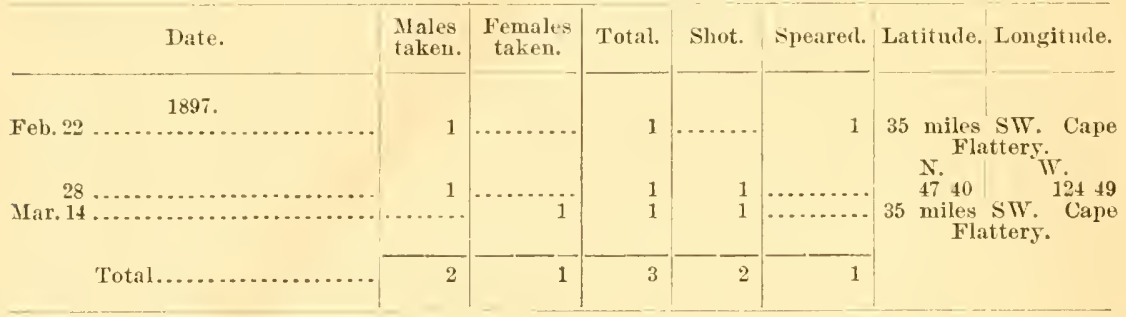

SEattle, Wash., Amil $2 S, 189 \%$.

Then personally appeared Jongie James, master of the within-named vessel, and made oath that the official log-book entries herein referred to, and any copies thereof herewith filed, and the answers to the foregoing questions made by him, are true, and the whole truth, to the best of his knowledge and belief.

Before me sworn to April 28, 1897.

\section{F. A. MCDONALD, Deputy Collector.}

Port of Port Townsend, Wash., Amil 30, $189 \%$.

I certify that the within is a true copy of the return on file in the customs office, Port of Port Townsend, and that the copy of the official log-book entries hereto annexed is a true cops.

\section{J. C. Saunders,}

Collector, Port of Poit Townsend.

ABSTRACT FROM THE SEAL LOG BOOK OF THE SCHOOXER JAMES G. SW AN ENGAGED IN FCR-SEAL FISHING IVNDER SPECIAL LICENSE ISSUED $\triangle T$ PORT ANGELES.

[Cleared from Port Angeles on Jan. 14, 1897; arrived from North Pacific Oceau on May 1, 1897; entered, at custom-house, Port Townseuch, on May 3, 1897.]

\begin{tabular}{|c|c|c|c|c|c|c|c|}
\hline Date. & $\begin{array}{l}\text { Males } \\
\text { titien. }\end{array}$ & $\begin{array}{c}\text { Females } \\
\text { taken. }\end{array}$ & Total. & shot. & speared. & Latitude. & Longitude. \\
\hline 1897 & & 1 & 1 & 1 & & N. & $\mathrm{II}^{2}$ \\
\hline 100. $27, \ldots \ldots \ldots$ & $i$ & 1 & 1 & 1 & & $\begin{array}{l}4130 \\
4148\end{array}$ & $\begin{array}{l}12455 \\
12450\end{array}$ \\
\hline Mar. $1 .$. & 1 & 9 & 10 & 8 & 2 & 4017 & 12453 \\
\hline $4 \ldots$ & ... & 2 & $\because$ & 1 & 1 & 3837 & $1 \geq 405$ \\
\hline $7 \ldots . .$. & ... & 1 & 1 & 1 & ..... & 3938 & 12437 \\
\hline $8 \ldots \ldots$ & -. & 1 & 1 & 1 & $\cdots$ & 4012 & 12500 \\
\hline $13 \ldots \ldots \ldots \ldots$ & 1 & $\begin{array}{c}6 \\
2 \\
2\end{array}$ & $?$ & $?$ & …....... & 4112 & 12449 \\
\hline Apr. $4 \ldots$. & & 2 & 2 & $\overline{2}$ & .............. & $\begin{array}{l}4112 \\
4752\end{array}$ & $\begin{array}{l}12454 \\
125 \quad 20\end{array}$ \\
\hline $9 . \ldots$ & 3 & 15 & 18 & 15 & 3 & 4736 & 12506 \\
\hline $12 \ldots \ldots \ldots$ & .......... & 5 & " & 5 & .......... & 4733 & 12500 \\
\hline 15. & & 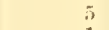 & 5 & 5 & ...... & 4839 & 12550 \\
\hline & ........ & 1 & 1 & 1 & .... & 4805 & 12556 \\
\hline T'otal. & 6 & 50 & 56 & 50 & 6 & & \\
\hline
\end{tabular}

P'ort Tow xsexd, May 3, 189\%.

Then personally appeared O. F. ('hristianson, master of the within-named ressel, and male oath that the official log-hook entries herein referrerl to, and any copies thereof herewith filed, and the answers to the foregoing questions made by him are true, and the whole truth, to the best of his knowlerge and belicf.

Before we,

Filed and recorded May $3,1897$.

Chas. Milder, Deputy Collector. 
Table C.-Log Entries of American Tessels Exgaged in Pelagic Sealing DURING TIIE SEASON OF 1897, ETC.-Continued.

Pont of Port Towssend, Wash., May $\delta, 18 \%$.

I certify that the within is a true copy of the return on file in the customs office, Port of l'ort Townsend, Wash., and that the copy of the official log-book entries hereto annexed is a true copy.

\section{J. C. SAUNders, \\ Collector, Port of P'ort Tounsend, Wash.}

ABSTRACT FROM THE SEAL LOG BOOK OF THE SCHOONER LENA L., ENGAGED IN FUR-SEAL FISHING UNDER SPECIAL LICENSE ISSUED AT SITKA, ALASKA.

Cleared from Sitka on Feb. 23, 1897; arrived from North Pacitic Ocean on May 22, 1897; entered at custom-house, Port Towusend, on May 22, 1897.]

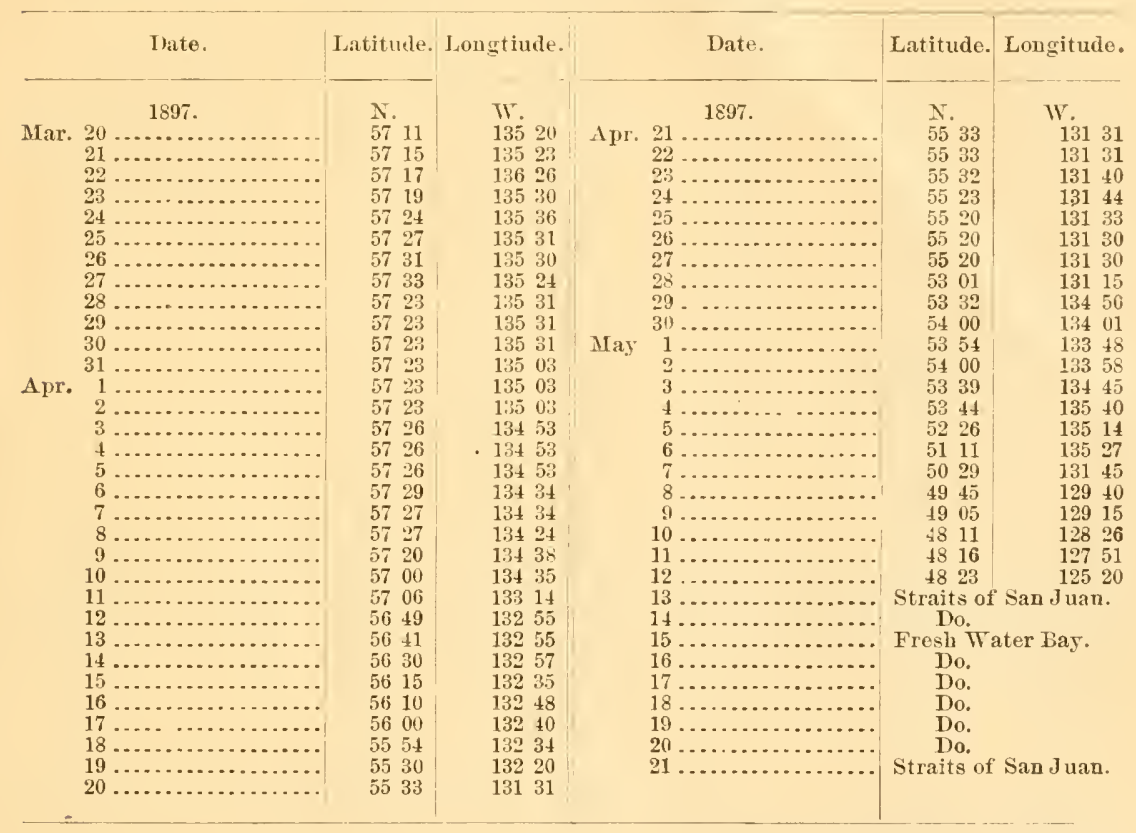

Port Townsend, May 22, 1897.

Then personally appeared Charles Prellberg, master of the within-llamed ressel, and made oath that the official log-book entries herein referred to, and any copies thereof herewith filed, and the answers to the foregoing questions made by him are true, anil the whole truth, to the best of his knowledge and belief.

Before me,

Filed and recorded May 22, 1897.

Chas. Miller, Deputy Collector.

Port Townsexd, WAsH., May $24,189:$.

I certify that the within is a true cops of the return on file in the customs office, Port of Port Townsend, ITash., and that the copy of the official log-book entries hereto annexed is a true cops.

J. C. SAUNDERS, Collector, Port of P'ort Townsend. 
Tamel C.--Log ENtries of Ankrican Vessels Extraged in Pelagic Sealing DURAg TII SE.ISON OF 1897, HTC.-Continued.

ABSTRACT FROM THE SEAL LOG BOOK OF 'THE SCIIOONER PUTITAN, ENGAGED IN FURSEAL FISHING UNDER SPECIAL LICENSE ISSE ED AT PORT ANGELES.

[Cleared from Port Angeles on Mar. 8, 1897; arrived from North Pacific Ocean on Apr. 27, 1897; entered at custon-house, Port Angeles, on A fr. 27, 1897, not having taken any seals.]

\begin{tabular}{|c|c|c|c|c|c|}
\hline & Date. & Latitude. Longitude. & Date. & Latitude. & Longitude. \\
\hline Apr. & 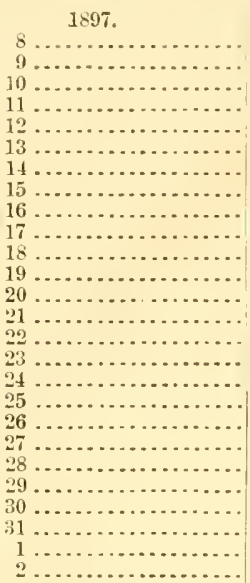 & $\begin{array}{l}\text { In Port Angeles. } \\
\text { In Crescent Bay. } \\
\text { Do. } \\
\text { In Jeah Bay. } \\
\text { Do. } \\
\text { Do. } \\
\text { Do. } \\
\text { Do. } \\
\text { Do. } \\
\text { Do. } \\
\text { Do. } \\
\text { Do. } \\
\text { Do. } \\
\text { Do. } \\
\text { Do. } \\
\text { Do. } \\
\text { Do. } \\
\text { Do. } \\
\text { Do. } \\
\text { Do. } \\
\text { Do. } \\
\text { Do. } \\
\text { Do. } \\
\text { Do. } \\
\text { Do. } \\
\text { Do. }\end{array}$ & 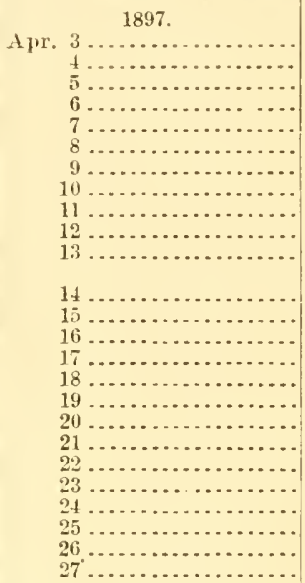 & 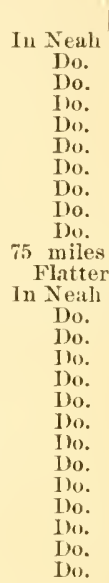 & $\begin{array}{l}\text { Bay. } \\
\text { ST. Capo } \\
\text { Sy. } \\
\text { Bay. }\end{array}$ \\
\hline
\end{tabular}

Custom House, Port ol Port Angelles, April 2\%, $189 \%$.

'Then personally appeared Irlis, master of the within-namerl vessel, and nuadeoath that the official log-book entries herein referred to, and any copies thereof herewith filed, and the answers to the foregoing questions nade by him are true, and the whole truth, to the best of his knowledge and belief.

Before me,

Wr. K. Clatik, Deputy Collector.

Poit of Port Tumnexd, Wasi., Amil 30, $189 \%$.

I certify that the within is a true copy of the return on file in the enstoms office, Port of Port Townsend, Wasl., and that the colyy of the official log-book entries hereto auncxed is a true copy.

\section{J. C. SAUNDERS,}

Collector, Port of Port Tounsend, II ash.

ABSTRACT FIOM THE SEAL LOG BOOK OF TIL SCHOONER ST. LAWRENCE, ENGAGED IN FTIS-SEAL FISIING UNDER SPEC1AL LICENSE ISSUED AT ATTU, ALASKA.

[C]eared from Seattle, Wash., on Dec. 28, 1896; arrived from Alaska on Oct.9, 1897; entered at customhouse, Port Townsend, on Oct. 9, 1s97.]

\begin{tabular}{|c|c|c|c|c|c|c|c|c|}
\hline & Date. & $\begin{array}{l}\text { Males } \\
\text { taken. }\end{array}$ & $\begin{array}{c}\text { Females } \\
\text { takinn. }\end{array}$ & Tutal. & Niliot. & Spealed. & Latitude. & Longitude. \\
\hline \multirow{6}{*}{ Mar. } & 1897 & & & & & & $\mathrm{~N}$ & E. \\
\hline & 16. & $\cdots$ & 9 & 9 & 9 & $\ldots$ & 3810 & 11151 \\
\hline & 18. & 1 & ... & 1 & 1 & & 3837 & 14226 \\
\hline & $\ldots$. & $\ldots$. & 1 & 1 & 1 & & $39 \quad 13$ & 14242 \\
\hline & $22 \ldots \ldots \ldots \ldots \ldots \ldots \ldots \ldots \ldots$ & + & 26 & 30 & 30 & -. & 3616 & 14146 \\
\hline & $30 \ldots \ldots \ldots \ldots \ldots \ldots \ldots \ldots$ & 1 & 3 & 4 & 4 & $\ldots \ldots \ldots$ & 3720 & 14225 \\
\hline \multirow[t]{3}{*}{ Apr. } & $2 \ldots \ldots \ldots \ldots \ldots \ldots$ & 8 & 41 & 49 & 49 & $\ldots \ldots . .$. & 3728 & $142 \quad 52$ \\
\hline & $5, \ldots \ldots \ldots \ldots \ldots \ldots \ldots \ldots$ & 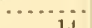 & 1 & 1 & 1 & $\ldots \ldots \ldots$ & 3714 & 14242 \\
\hline & ${ }_{1}{ }_{1}{ }_{1}{ }_{1}$ & $\begin{array}{l}14 \\
\ldots\end{array}$ & $\begin{array}{r}82 \\
2\end{array}$ & $\begin{array}{r}96 \\
2\end{array}$ & $\begin{array}{r}96 \\
2\end{array}$ & . $\ldots \ldots \ldots$ & $\begin{array}{ll}37 & 31 \\
37 & 35\end{array}$ & 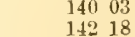 \\
\hline
\end{tabular}


Table C.-Log Entrieg of American Tessels Engaged in Pelagic Sealing DURING THE SEASON OF 1897 , ETC.-Contimued.

ABSTRACT FROM THE SEAL LOG BOOK OF THE SCHOONER ST. LAWßENCE, E'c.-Continned.

\begin{tabular}{|c|c|c|c|c|c|c|c|c|}
\hline & Date. & $\begin{array}{l}\text { Males } \\
\text { taken. }\end{array}$ & $\begin{array}{c}\text { Females } \\
\text { taken. }\end{array}$ & Total. & Shot. & Speared. & Latitude. & Longitude. \\
\hline \multirow{15}{*}{$\begin{array}{l}1 \\
1 \\
1 \\
1 \\
1 \\
1 \\
\\
\\
\\
\\
3 \\
3\end{array}$} & 1897 & & & & & & N. & E. \\
\hline & 8 & $\ddot{8}$ & 1 & 1 & 1 & $\cdots$ & 3748 & 14218 \\
\hline & $\begin{array}{r}9 \\
11\end{array}$ & & 17 & 25 & 95 & $\ldots \ldots \ldots$ & 3705 & 14225 \\
\hline & $12 \ldots \ldots \ldots \ldots \ldots \ldots$ & $\ldots \ldots \ldots$ & 1 & 1 & 1 & $\ldots \ldots \ldots$ & 3746 & 14218 \\
\hline & $13 \ldots \ldots \ldots \ldots \ldots \ldots$ & 9 & $\begin{array}{r}4 \\
13\end{array}$ & $\begin{array}{r}4 \\
29\end{array}$ & $\begin{array}{r}4 \\
22\end{array}$ & ........... & 3726 & 14130 \\
\hline & $15 \ldots \ldots \ldots \ldots \ldots \ldots \ldots \ldots \ldots$ & 1 & $\begin{array}{c}13 \\
\cdots \\
\cdots\end{array}$ & 1 & 1 & $\cdots \ldots \ldots \ldots$ & 3841 & $\begin{array}{l}11148 \\
14239\end{array}$ \\
\hline & $17 \ldots \ldots \ldots \ldots \ldots \ldots \ldots$ & 2 & 15 & 17 & 17 & $\ldots \ldots$. & 3752 & $144 \quad 46$ \\
\hline & $19 \ldots \ldots \ldots \ldots \ldots \ldots \ldots \ldots \ldots \ldots \ldots$ & $\ldots \ldots$ & 1 & 1 & 1 & $\ldots \ldots \ldots$ & 3820 & 14133 \\
\hline & $20 \ldots \ldots \ldots \ldots \ldots \ldots \ldots \ldots$ & 11 & 21 & 32 & 32 & $\ldots \ldots \ldots$ & 3809 & 14212 \\
\hline & $21, \ldots \ldots \ldots \ldots \ldots \ldots \ldots \ldots \ldots \ldots \ldots$ & ........ & 1 & 1 & 1 & .......... & 3830 & 14203 \\
\hline & & 1 & .......... & 1 & 1 & $\ldots \ldots \ldots$ & 3830 & 14220 \\
\hline & $\begin{array}{l}26 \\
27\end{array}$ & $\cdots$ & $\begin{array}{l}10 \\
20\end{array}$ & 10 & 10 & (......... & $380 \pm$ & 14220 \\
\hline & & $\begin{array}{l}2 \\
1\end{array}$ & $\begin{array}{r}20 \\
3\end{array}$ & 22 & 22 & $\ldots \ldots \ldots$. & $37+5$ & 14154 \\
\hline & 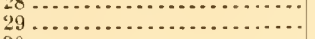 & 8 & $\begin{array}{r}3 \\
26\end{array}$ & $\begin{array}{r}4 \\
34\end{array}$ & 4 & $\ldots$ & 3720 & 14202 \\
\hline & $30 \ldots \ldots \ldots \ldots \ldots \ldots \ldots \ldots \ldots$ & 2 & 20 & $\begin{array}{r}34 \\
2\end{array}$ & $\begin{array}{r}34 \\
2\end{array}$ & $\ldots \ldots \ldots$ & $\begin{array}{l}3825 \\
3807\end{array}$ & 14357 \\
\hline \multirow[t]{12}{*}{ May } & $1 \ldots \ldots \ldots \ldots \ldots$ & 1 & 4 & 5 & 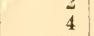 & $\cdots \cdots$ & 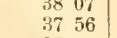 & $\begin{array}{l}14343 \\
14359\end{array}$ \\
\hline & $2 \ldots \ldots \ldots \ldots \ldots \ldots \ldots \ldots \ldots \ldots \ldots \ldots \ldots \ldots$ & 12 & 39 & 51 & 51 & $\ldots \ldots \ldots$ & 3743 & 14327 \\
\hline & $3 \ldots \ldots \ldots \ldots \ldots \ldots \ldots \ldots$ & 3 & 30 & 33 & 33 & .......... & 3746 & $14: 330$ \\
\hline & $10 \ldots \ldots \ldots \ldots \ldots \ldots$ & $\ldots \ldots \ldots$ & 5 & 5 & 5 & $\ldots \ldots \ldots$ & 4117 & 14229 \\
\hline & $11 \ldots \ldots \ldots \ldots \ldots$ & 1 & 10 & 11 & 11 & ............ & 4143 & 14213 \\
\hline & $12 \ldots \ldots \ldots \ldots$ & $\because$ & 8 & 8 & 8 & .......... & 4150 & 14211 \\
\hline & $13 \ldots \ldots \ldots \ldots \ldots \ldots \ldots \ldots$ & 4 & 8 & 12 & 12 & . $\ldots \ldots \ldots$ & 4152 & 14213 \\
\hline & $14 \ldots \ldots \ldots \ldots \ldots \ldots$ & 1 & 2 & 3 & 3 & .......... & \multicolumn{2}{|c|}{ Straits of Tsugar. } \\
\hline & & 2 & $\begin{array}{l}6 \\
2\end{array}$ & 8 & 8 & .......... & \multirow{2}{*}{\multicolumn{2}{|c|}{$\begin{array}{l}\text { Hakorlate. } \\
\text { Tsugar Straits. }\end{array}$}} \\
\hline & $\begin{array}{r}23 \\
24 \\
24\end{array}$ & $\begin{array}{l}3 \\
8\end{array}$ & $\frac{2}{7}$ & 5 & 5 & (......... & & \\
\hline & & $\frac{8}{5}$ & 6 & 15 & 15 & (.......... & Di. & \\
\hline & & $\begin{array}{l}3 \\
5\end{array}$ & $\begin{array}{l}6 \\
2\end{array}$ & 11 & 11 & 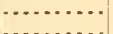 & Do. & \\
\hline \multirow[t]{8}{*}{ June } & $2 \ldots \ldots \ldots \ldots \ldots$ & 1 & 2 & $\begin{array}{l}7 \\
1\end{array}$ & 7 & $\ldots \ldots \ldots$ & Do. & \\
\hline & $4 \ldots \ldots$ & 5 & 13 & $\begin{array}{r}1 \\
18\end{array}$ & $\begin{array}{r}1 \\
18\end{array}$ & ( & 4246 & 14657 \\
\hline & $5 \ldots \ldots \ldots \ldots \ldots \ldots$ & 1 & 9 & $\begin{array}{l}18 \\
10\end{array}$ & $\begin{array}{l}18 \\
10\end{array}$ & $\cdots \cdots$ & $\begin{array}{l}43 \quad 26 \\
43 \quad 27\end{array}$ & 14654 \\
\hline & $6 \ldots \ldots \ldots \ldots \ldots \ldots$ & 1 & 1 & $\begin{array}{r}10 \\
2\end{array}$ & $\begin{array}{r}10 \\
2\end{array}$ & (n......... & $\begin{array}{l}4327 \\
4322\end{array}$ & \\
\hline & $9 \ldots \ldots \ldots \ldots$ & 4 & $\ldots$ & 4 & $\dot{4}$ & ........ & 4310 & $\begin{array}{ll}147 & 42 \\
146 & 00\end{array}$ \\
\hline & $10 \ldots \ldots \ldots \ldots \ldots \ldots$ & 7 & 1 & 8 & 8 & .......... & 4320 & $146 \quad 16$ \\
\hline & $21, \ldots \ldots \ldots$ & $\cdots$ & 1 & 1 & 1 & $\ldots .$. & $46 \quad 32$ & 14644 \\
\hline & $250 \ldots+\ldots$ & $\cdots$ & 4 & 4 & 4 & $\ldots \ldots \ldots$ & 5020 & 15605 \\
\hline July & 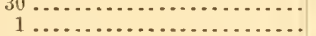 & $\begin{array}{l}1 \\
4\end{array}$ & 47 & 1 & 1 & ......... & 5035 & 15612 \\
\hline & $3 \ldots \ldots$ & $\begin{array}{l}+ \\
\cdots\end{array}$ & $\begin{array}{r}47 \\
3\end{array}$ & 51 & 51 & ........... & $\begin{array}{ll}50 & 05 \\
5 & 09\end{array}$ & 15602 \\
\hline & $4 \ldots \ldots \ldots \ldots \ldots \ldots \ldots \ldots$ & 2 & 1 & $\begin{array}{l}3 \\
3\end{array}$ & $\begin{array}{l}3 \\
3\end{array}$ & $\ldots \ldots \ldots$ & $\begin{array}{l}5002 \\
4955\end{array}$ & $\begin{array}{l}14606 \\
146 \quad 15\end{array}$ \\
\hline & $5 \ldots \ldots \ldots$ & $\cdots$ & 9 & 9 & 9 & ......... & 5007 & $1+602$ \\
\hline & .............. & . & 1 & 1 & 1 & 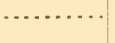 & $\begin{array}{l}4.907 \\
\text { N. }\end{array}$ & $\prod^{155} 31$ \\
\hline \multirow[t]{17}{*}{ Aug. } & $2 \ldots \ldots \ldots \ldots \ldots \ldots \ldots$ & ........ & 6 & 6 & $\cdots$. & 6 & 5540 & 16707 \\
\hline & $6 \ldots \ldots \ldots \ldots$ & $\ldots \ldots$. & 3 & 3 & $\ldots$ & 3 & 5723 & 16625 \\
\hline & $7 \ldots \ldots \ldots \ldots \ldots \ldots \ldots \ldots \ldots \ldots$ & $\cdots$ & 4 & 4 & $\ldots$. & 4 & 5640 & 16620 \\
\hline & 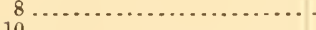 & $\cdots$ & 1 & 1 & ......... & 1 & 5620 & 16710 \\
\hline & $10 \ldots \ldots+\ldots \ldots \ldots$ & 6 & 7 & 13 & $\ldots \ldots \ldots$ & 13 & 5520 & 16830 \\
\hline & $\begin{array}{l}11 \\
12 \\
12 \ldots \ldots\end{array}$ & $\begin{array}{l}1 \\
4\end{array}$ & 8 & 1 & $\cdots \ldots \ldots$ & 1 & 5516 & 16816 \\
\hline & 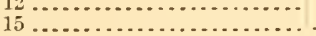 & $\begin{array}{l}4 \\
\cdots\end{array}$ & $\begin{array}{r}8 \\
11\end{array}$ & 12 & - . n...... & 12 & 5515 & 16850 \\
\hline & $16 \ldots \ldots \ldots \ldots$ & 1 & $\begin{array}{r}11 \\
4\end{array}$ & 11 & ….... & 11 & 5621 & 16653 \\
\hline & $18 \ldots \ldots \ldots \ldots \ldots \ldots \ldots$ & 1 & $\begin{array}{r}4 \\
17\end{array}$ & 5 & $\ldots \ldots \ldots$ & $\begin{array}{r}5 \\
18\end{array}$ & 5609 & 16656 \\
\hline & 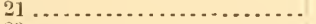 & 3 & 14 & $\begin{array}{l}18 \\
17\end{array}$ & $\ldots \ldots \ldots$ & $\begin{array}{l}18 \\
17\end{array}$ & 5522 & 16928 \\
\hline & $23 \ldots \ldots \ldots \ldots \ldots \ldots \ldots \ldots \ldots \ldots \ldots$ & . & 4 & 4 & ( & $\begin{array}{r}17 \\
4\end{array}$ & $\begin{array}{ll}55 & 12 \\
55 & 17\end{array}$ & $\begin{array}{l}16931 \\
168 \quad 52\end{array}$ \\
\hline & $24 \ldots \ldots \ldots \ldots$ & 3 & 5 & 8 & ......... & 8 & 5448 & 16530 \\
\hline & 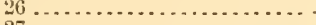 & $=$ & 1 & 1 & $\ldots \ldots$. & 1 & 5510 & 16801 \\
\hline & $27 \ldots \ldots \ldots \ldots$ & 7 & 17 & 24 & ....... & 24 & 5505 & 16855 \\
\hline & 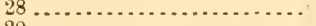 & 3 & 5 & 8 & $\ldots \ldots .$. & 8 & 5505 & 16902 \\
\hline & $99 \ldots \ldots \ldots \ldots \ldots \ldots \ldots \ldots$ & 2 & 9 & 11 & ........ & 11 & $55 \quad 07$ & 16909 \\
\hline & 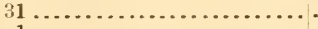 & $\cdots$ & 5 & 5 & $\ldots \ldots \ldots$ & 5 & 5517 & 16027 \\
\hline \multirow[t]{7}{*}{ Sept. } & $1 \ldots \ldots \ldots \ldots \ldots \ldots \ldots$ & 2 & 7 & 9 & $\ldots \ldots$. & 9 & 5508 & $168 \quad 18$ \\
\hline & $2 \ldots \ldots \ldots \ldots \ldots \ldots \ldots$ & 3 & 6 & 9 & $\ldots \ldots$. & 9 & 5446 & 16839 \\
\hline & $3 \ldots \ldots \ldots \ldots \ldots$ & 6 & 16 & 22 & ......... & 22 & 5505 & 16822 \\
\hline & 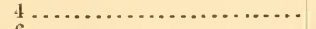 & 1 & 1 & 2 & ....... & 2 & 5500 & 16817 \\
\hline & $6 \ldots \ldots \ldots \ldots \ldots \ldots$ & 1 & $\because$ & 1 & ......... & 1 & $5 j 17$ & 16840 \\
\hline & $11 \ldots \ldots \ldots \ldots \ldots \ldots \ldots$ & 1 & 3 & 4 & (........ & 4 & 5427 & 16705 \\
\hline & 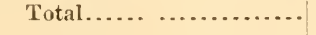 & 189 & 671 & 860 & 660 & 200 & & \\
\hline
\end{tabular}


Table C.- Tog Entries of American Tessels Exgafen in Pleagic Sealixg DUling THE SEasox of 1897, ETC.-Continued.

Tessels landing their catches at San Francisco.

\begin{tabular}{|c|c|c|c|c|c|}
\hline Name. & Males. & Females. & Total. & Shot. & Spearell. \\
\hline 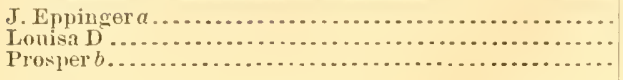 & $\begin{array}{r}140 \\
93 \\
85\end{array}$ & $\begin{array}{l}945 \\
636 \\
139\end{array}$ & $\begin{array}{r}1,085 \\
729 \\
224\end{array}$ & $\begin{array}{l}642 \\
729 \\
224\end{array}$ & $\begin{array}{r}443 \\
\ldots \ldots \ldots \\
\ldots \ldots \ldots\end{array}$ \\
\hline 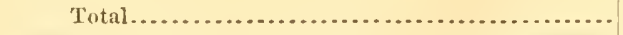 & 318 & 1,720 & 2,038 & 1,595 & 413 \\
\hline
\end{tabular}

a The Eppinger took 319 of these skins, all females, south of the award area.

$b$ The entire cateh of the Prosper was taken south of the award area, eIose otf' the GaJapagos Islands.

ABSTRACT FROM THE SEAL LOG BOOK OF THE SCHOONER J. EPPINGER, ENGAGED

IN FUR-SEAL FISHING UNIER SPECIAL LICENSE ISSUED AT SAN FRANCISCO.

[Cleared from San Franeiseo Dee. 3, 1896; arrived from sealing royage on Mar. 28, 1897; entered at custom-house, San Francisco, on Mar. 30, 1897.]

\begin{tabular}{|c|c|c|c|c|c|c|c|}
\hline & Date. & $\begin{array}{l}\text { Dlakes } \\
\text { taken. }\end{array}$ & $\begin{array}{c}\text { Females } \\
\text { talien. }\end{array}$ & Total. & Shot. & Latitude. & Longitude. \\
\hline \multirow{12}{*}{ Dec. } & 1896. & & & & & & \\
\hline & (n. & & & $\cdots$ & $\cdots$ & 3735 & $123 \quad 40$ \\
\hline & & & & & $\cdots$ & ( W....... & $\cdots+\cdots$ \\
\hline & & & $\begin{array}{l}\cdots \\
\cdots\end{array}$ & $\begin{array}{ll}\cdots \\
\cdots\end{array}$ & & (......... & (n) \\
\hline & 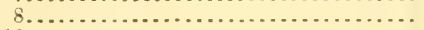 & & .. & $\ldots$ & & 3310 & 12035 \\
\hline & ................. & & 1 & 1 & 1 & 3325 & 12040 \\
\hline & .................. & & 5 & 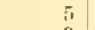 & 5 & 3757 & 12322 \\
\hline & $\ldots \ldots \ldots \ldots \ldots \ldots$ & -.. & 3 & 3 & 3 & 3749 & 12435 \\
\hline & $\ldots \ldots \ldots \ldots \ldots \ldots$ & $\ldots$ & 3 & 3 & 3 & 3756 & 12411 \\
\hline & . & ... & 9 & 9 & 9 & 3746 & 12446 \\
\hline & $\ldots \ldots \ldots \ldots \ldots \ldots . . . . . .$. & $\cdots$ & 4 & 4 & 4 & 3754 & 12406 \\
\hline & 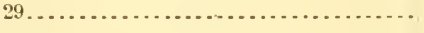 & $\cdots$ & 12 & 12 & 12 & 3726 & 12325 \\
\hline \multirow{20}{*}{ Jan. } & 1897. & & & & & & \\
\hline & . & $\ldots$ & 8 & 8 & 8 & 3755 & 12315 \\
\hline & $\ldots \ldots \ldots \ldots \ldots \ldots$ & ... & 1 & 1 & 1 & 3736 & 12315 \\
\hline & ...... & $\cdots$ & 20 & 20 & 20 & 3731 & 12346 \\
\hline & ............ & $\ldots \ldots \ldots$ & 22 & 22 & 22 & 3715 & $12+25$ \\
\hline & $7 \ldots \ldots \ldots \ldots \ldots$ & ........ & 3 & 3 & 3 & 3717 & 12415 \\
\hline & 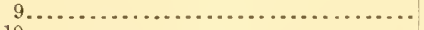 & 2 & 19 & 21 & 21 & 3712 & 12425 \\
\hline & 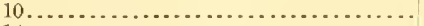 & ........ & 16 & 16 & 16 & 3714 & 12125 \\
\hline & 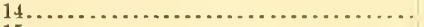 & ........ & 4 & 4 & 4 & 3626 & 12256 \\
\hline & 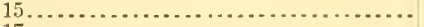 & ......... & 2 & 2 & 2 & 3601 & 12210 \\
\hline & 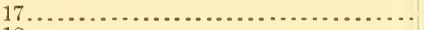 & 2 & 10 & 12 & $1 \overline{2}$ & 3644 & 12227 \\
\hline & 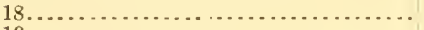 & ........ & 26 & 26 & 26 & 3650 & 12241 \\
\hline & 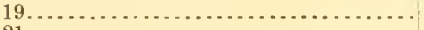 & (........ & 24 & 24 & 24 & 3654 & 12258 \\
\hline & 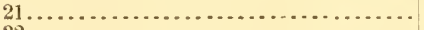 & ........ & 4 & 4 & 4 & 3615 & 12210 \\
\hline & 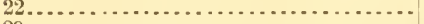 & ......... & 7 & 7 & 7 & $\begin{array}{lll}36 & 12\end{array}$ & 12200 \\
\hline & 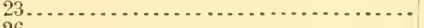 & ........ & 9 & 9 & 9 & $36 \quad 29$ & 12230 \\
\hline & 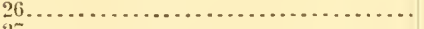 & ......... & 5 & 5 & 5 & 3534 & 12123 \\
\hline & (., & - & 2 & 2 & 2 & & \\
\hline & (n) & ......... & 52 & 52 & 52 & 3442 & 12050 \\
\hline & 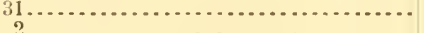 & ........ & & $\cdots$ & & DR.34 40 & 12200 \\
\hline \multirow[t]{14}{*}{ Feb. } & 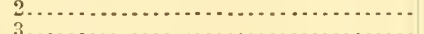 & ........ & 28 & 28 & 28 & 3457 & 12050 \\
\hline & 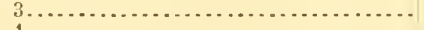 & $\ldots \ldots \ldots$ & 12 & 12 & 12 & 3447 & 12147 \\
\hline & (n, & ........ & \pm 2 & 42 & 42 & 3434 & 12046 \\
\hline & (1) & $\ldots \ldots$ & 6 & 6 & 6 & 3448 & 12050 \\
\hline & $\begin{array}{r}9 \\
14 \ldots \ldots\end{array}$ & $\ldots \ldots \ldots$ & 1 & 1 & 1 & $\ldots \ldots$ & $\ldots \ldots$ \\
\hline & & ........ & 13 & 13 & 13 & .......... & $\ldots$ \\
\hline & $15{ }_{10}$ & $\ldots \ldots$ & 27 & 27 & 27 & 3110 & 11930 \\
\hline & & . $\ldots \ldots$ & 5 & 5 & 5 & 1 $\ldots \ldots \ldots$ & $190=0$ \\
\hline & & $\ldots \ldots$ & 33 & 33 & 33 & 3433 & $120 \quad 59$ \\
\hline & & ........ & 25 & 25 & 25 & 3435 & 12045 \\
\hline & (n) & $\ldots$ & 13 & 13 & 13 & 3436 & 12034 \\
\hline & (n. & $\cdots$ & 2 & 2 & 2 & 3440 & $121 \quad 15$ \\
\hline & ..... & $\cdots$ & $\stackrel{2}{0}$ & 2 & 2 & 3455 & 12040 \\
\hline & ........ & $\cdots$ & 28 & 28 & 28 & $3 \pm 56$ & 12038 \\
\hline \multirow[t]{14}{*}{ Mar. } & (n...... & $\cdots$ & 8 & 8 & 8 & 3540 & 12136 \\
\hline & $\begin{array}{ll}\ldots \ldots \\
\cdots \ldots\end{array}$ & $\cdots$ & 1 & 1 & 1 & 3522 & 1:2135 \\
\hline & 5. & $\ldots$ & 7 & 7 & 7 & 3145 & 12059 \\
\hline & ....... & ... & 2 & 2 & 2 & 3421 & 12000 \\
\hline & 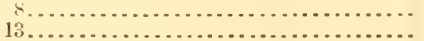 & $\cdots$ & 20 & 20 & 20 & 3411 & 12047 \\
\hline & (1, & $\because$ & 13 & 13 & 13 & 3507 & $121=8$ \\
\hline & (n. & 1 & 5 & 6 & 6 & $\begin{array}{ll}35 & 23 \\
\end{array}$ & 12158 \\
\hline & 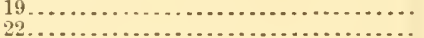 & $\cdots$ & 2 & 2 & 2 & 36.37 & 12221 \\
\hline & (n) & . & 1 & 1 & 1 & 3635 & 12220 \\
\hline & $\ldots \ldots$ & - - & 38 & 38 & 38 & 3655 & 12230 \\
\hline & n. & 1 & 7 & 8 & 8 & 3650 & 12232 \\
\hline & (n., & 1 & 22 & 23 & 23 & 3655 & 12245 \\
\hline & $27 \ldots \ldots \ldots \ldots$ & & 1 & 1 & 1 & 3654 & 12245 \\
\hline & Total ........ & 7 & 635 & 642 & 612 & & \\
\hline
\end{tabular}


Table C.-Log Entries of American Tessels Engaged in Pelagic Sealing DERING THE SEASON OF 1897, ETC.-Continued.

\section{DISTRICT ANI POIT OF SAN FRANCISCO.}

I, M. White, master of the American schooner J. Eppinger, do solemnly and truly swear that the foregoing abstract from the seal $\log$ book of the said vessel is correct; that the number and sex of seals taken on the days specified, with the latitude and longitude, and all other particulars as herein expressed, are true to the best of my knowledge and belicf. So help me God.

Subscribed and sworn to before me this 30 th day of March, 1897.

M. WHITE, Master.

[STAL.]

N. S. FARLEY,

Deputy Collector of Customs.

\section{Custon-HousE,}

San Francisco, April 1, 1897.

I herelyy certify that the foregoing is a true copy of the original extract from the official seal $\log$ book of the American schooner $\vec{J}$. Eppinger, and that said official seal log and the original extract therefrom were presented and filed in this office on March 30, I897.

\section{N. S. FARLEY, Ireputy Cullector of Customs.}

ABSTRACT FROM THE SEAL LOG BOOK OF THE SCHOONER J. EPPINGER, ENGAGED IN FUR-SEAL FISHING UNDER SPECLAL LICENSE ISSUED AT UNALASKA.

[Cleared from Unalaska on July 29, 1897; arrived from Unaliska on Sept. 30, 1897; entered at custom. house, San Francisco, on Oct. 2, 1897.]

\begin{tabular}{|c|c|c|c|c|c|c|c|}
\hline & Date. & $\begin{array}{l}\text { Males } \\
\text { taken. }\end{array}$ & $\begin{array}{c}\text { Females } \\
\text { taken. }\end{array}$ & Total. & speared. & Latitude. & Longitude. \\
\hline Sept. & 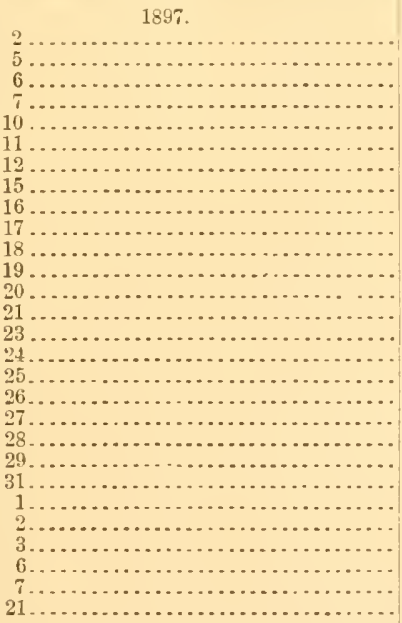 & $\begin{array}{rr}5 \\
5 \\
1 \\
3 \\
3 \\
9 \\
1 \\
3 \\
3 \\
\ldots \\
6 \\
9 \\
3 \\
7 \\
9 \\
6 \\
20 \\
2 \\
1 \\
6 \\
6 \\
16 \\
3 \\
5 \\
1 \\
\ldots . .\end{array}$ & $\begin{array}{r}2 \\
2 \\
21 \\
11 \\
5 \\
12 \\
11 \\
15 \\
2 \\
5 \\
15 \\
1 \\
2 \\
16 \\
12 \\
38 \\
24 \\
15 \\
52 \\
10 \\
4 \\
7 \\
5 \\
7 \\
2 \\
12 \\
2 \\
\ldots \\
\ldots\end{array}$ & $\begin{array}{r}2 \\
2 \\
26 \\
16 \\
6 \\
15 \\
14 \\
24 \\
3 \\
8 \\
18 \\
1 \\
8 \\
25 \\
15 \\
45 \\
33 \\
21 \\
72 \\
12 \\
5 \\
13 \\
11 \\
23 \\
5 \\
17 \\
3 \\
\ldots \ldots\end{array}$ & $\begin{array}{r}2 \\
2 \\
26 \\
16 \\
6 \\
15 \\
14 \\
24 \\
3 \\
8 \\
18 \\
1 \\
8 \\
25 \\
15 \\
45 \\
33 \\
21 \\
72 \\
12 \\
5 \\
13 \\
11 \\
23 \\
5 \\
17 \\
3 \\
\ldots \ldots\end{array}$ & \begin{tabular}{|ll}
5 & 19 \\
55 & 00 \\
55 & 00 \\
54 & 54 \\
55 & 45 \\
55 & 42 \\
55 & 48 \\
55 & 25 \\
55 & 20 \\
55 & 13 \\
55 & 16 \\
55 & 04 \\
54 & 50 \\
54 & 57 \\
55 & 11 \\
55 & 16 \\
55 & 15 \\
55 & 10 \\
54 & 50 \\
54 & 40 \\
55 & 18 \\
55 & 08 \\
55 & 17 \\
55 & 05 \\
54 & 41 \\
55 & 09 \\
55 & 05 \\
DR.45 & 36
\end{tabular} & $\begin{array}{ll}\text { W. } & \\
167 & 15 \\
168 & 53 \\
168 & 45 \\
168 & 45 \\
171 & 05 \\
171 & 51 \\
171 & 52 \\
170 & 38 \\
170 & 45 \\
170 & 51 \\
170 & 00 \\
168 & 40 \\
168 & 20 \\
168 & 20 \\
169 & 20 \\
169 & 21 \\
169 & 30 \\
169 & 40 \\
169 & 48 \\
169 & 40 \\
169 & 11 \\
169 & 27 \\
169 & 59 \\
169 & 04 \\
169 & 00 \\
169 & 47 \\
169 & 37 \\
147 & 53\end{array}$ \\
\hline & Total ....... & 133 & 310 & 443 & 443 & & \\
\hline
\end{tabular}

District and Port of San Francisco.

I, M. White, master of the American schooner $J$. Eppinger, do solemuly and truly swear that the folegoing abstract from the seal $\log$ book of said vessel is correct; that the number and sex of seals taken on the days specified, with the latitude and longitude, and all other particulars as herein expressed, are true to the best of my knowlerige and belief, so help me God.

Suliscribed and sworn to before me this 2 day of October, 1897.

M. WIITE, Master.

N. S. FARLEY,

l)eputy Collector of Customs. 
Table C.-Log Entries of Aliejican Vessels ENgaged in Pelagic Sealing DURING TIE SEASON Ol 1897, ETC.-Continued.

Custom-House, San Francisco, October 4, 1897.

I hereby certify that the foregoing is a true coly of the original abstract from the official seal $\log$ of the American schooner $J$. Eppinger; that the said original abstract has been compared with the official seal $\log$ and found to be correct; and that said original abstract and official seal $\log$ are on file in this office.

N. S. FARIEY,

Deputy Collector of c'ustoms.

ABSTRACT FROM THE SEAL LOG BOOK OF THE AMERICAN SCHOONER LOUISA D., ENGAGED IN FUR-SEAL FISHING UNDER SPECIAL LICWNSE ISSUED AT SAN FRAN. CISCO DECEMBER 12, 1896.

[Cleared from San Francisco on Dec. 14, 1896; arrived from sea on Apr. 27, 1897; entered at custom. honse, san Francisco, on A pr. 27, 1897.

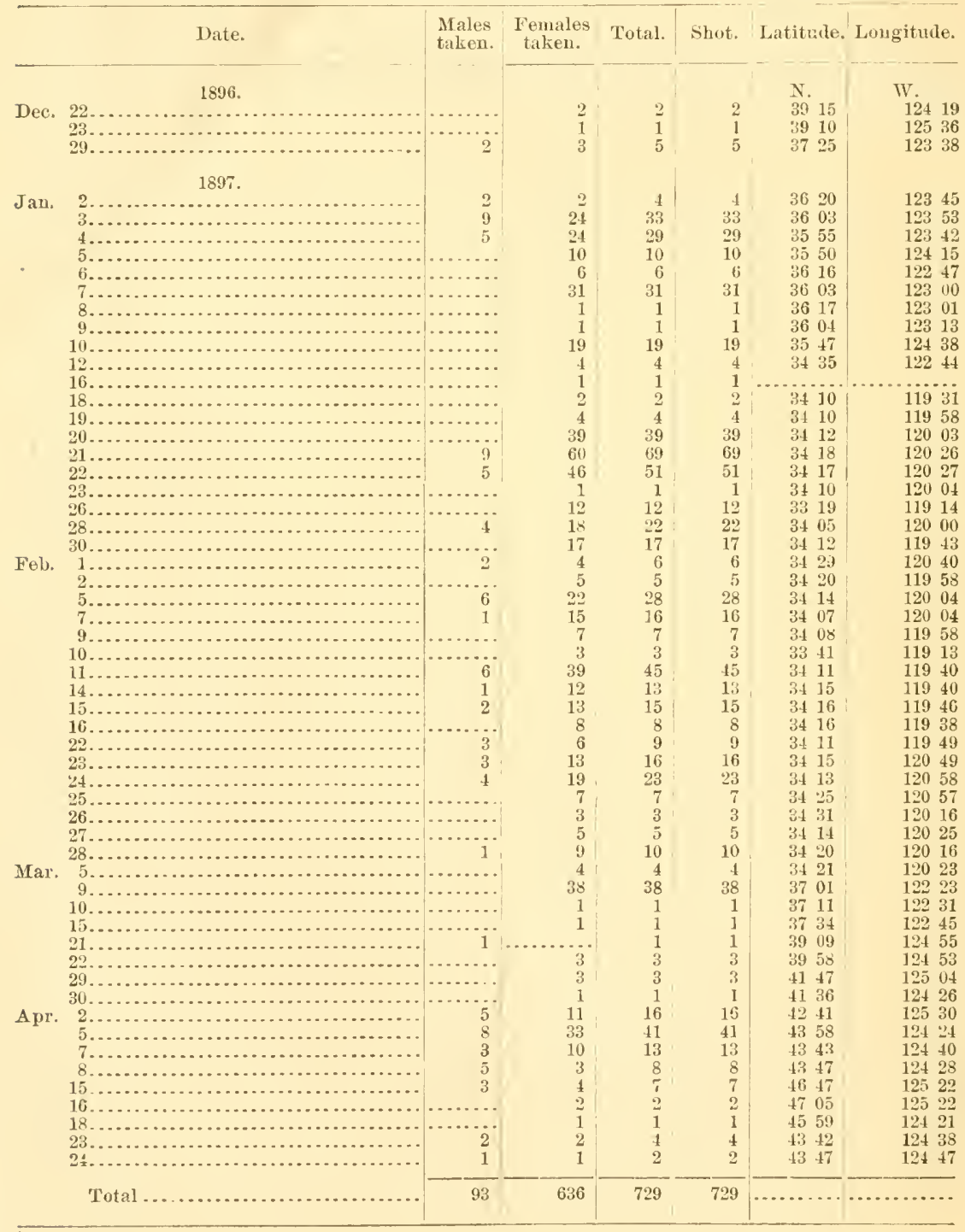


Table C.-Log Entries of Americax V́essels Exgaged in Pelagic Shalixg DURING THE SEASON OF 1897, ETC.-C'ontinlled.

District ani Port of San Francisco.

I, S. H. Burtis, master of the American schomer Lonisa I., do solemnly and truly swear that the foregoing abstract from the seal $\log$ look of the said vessel is correct; that the number anil sex of seals taken on the dass specified, with the latitude and longitude, and all other particulars as herein expressed, are true to the best of my knowledge and belief. So help me God.

S. H. Burtis.

Subseribed and sworn to before me this 28th day of April, 1897.

N. S. Farter, Deputy Collector of Customs.

Ciston-Hoese, San Francisco, April $30,189 \%$.

I hereby certify that the foregoing is a true copy of the original extract from the official seal $\log$ of the American schooner Louisa. $\mathrm{D}$., which vessel arrived from a fursealing voyage April 27, 1897. I further certity that the oftirial seal $\log$ and the original extract therefrom were tiled in this office April 28, 1897.

[SEAL.]

N. S. FarLEY, Deputy Collector of Customs.

ABSTRACT FROM THE SEAL LOG BOOK OF THE SCHOOXER PROSPER, ENGAGED IN FIR-SEAL FISHING UNDER SPECIAL LICENSE ISSUED AT SAN FRANCISCO.

[Cleared from San Francisco on Apr. 30, 1897, arriced on Dec. 14, 1897, entered at custom-house. San Francisco, on Dec. 15, 1897.]

\begin{tabular}{|c|c|c|c|c|c|c|c|}
\hline & Date. & $\begin{array}{l}\text { Males } \\
\text { taken. }\end{array}$ & $\begin{array}{c}\text { Females } \\
\text { taken. }\end{array}$ & Total. & Shot. & Latitude. & Longitude. \\
\hline \multirow{5}{*}{ July } & 1897. & & & & & $\mathrm{~N}$. & WV. \\
\hline & 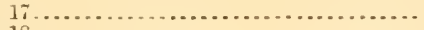 & 5 & 5 & 10 & 10 & 123 & 9150 \\
\hline & 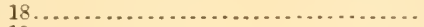 & 2 & 4 & 6 & 6 & 123 & 9150 \\
\hline & $19 \ldots \ldots \ldots \ldots$ & 3 & 8 & 11 & 11 & 123 & 9150 \\
\hline & 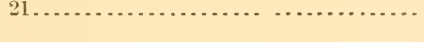 & 5 & 8 & 13 & 13 & $\begin{array}{l}126 \\
\text { s. }\end{array}$ & 9150 \\
\hline \multirow[t]{8}{*}{ Aug. } & 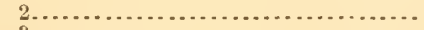 & 0 & 1 & 1 & 1 & 013 & 9136 \\
\hline & $3 \ldots \ldots \ldots \ldots \ldots \ldots \ldots \ldots \ldots \ldots \ldots \ldots \ldots \ldots$ & 3 & 13 & 16 & 16 & 013 & 9135 \\
\hline & 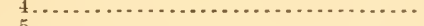 & 9 & 7 & 16 & 16 & 017 & 9140 \\
\hline & 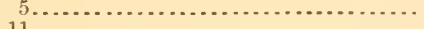 & 1 & 1 & 2 & 2 & 015 & 9140 \\
\hline & & 7 & 9 & 16 & 16 & 100 & 9130 \\
\hline & 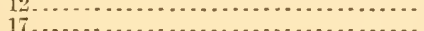 & $\begin{array}{l}4 \\
0\end{array}$ & 12 & 16 & 16 & $\begin{array}{l}100 \\
100\end{array}$ & 9130 \\
\hline & 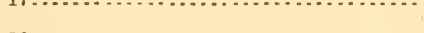 & 0 & i & 3 & 3 & N. & 9140 \\
\hline & 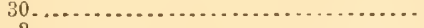 & 3 & 3 & 6 & 6 & 020 & 9000 \\
\hline \multirow[t]{20}{*}{ Sept. } & $2 \ldots \ldots \ldots \ldots \ldots \ldots$ & 3 & 2 & 5 & 5 & $\begin{array}{r}020 \\
\text { S. }\end{array}$ & 9000 \\
\hline & $6 \ldots \ldots$ & 2 & 10 & 12 & 12 & () 17 & 9126 \\
\hline & 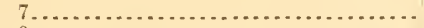 & 2 & 2 & 4 & 4 & 019 & 9126 \\
\hline & 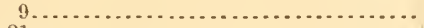 & $\overrightarrow{1}$ & 0 & 1 & 1 & 016 & 9140 \\
\hline & 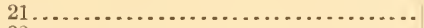 & 3 & 3 & 6 & 6 & 100 & 9100 \\
\hline & 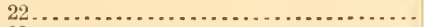 & 2 & 7 & 9 & 9 & 050 & 9125 \\
\hline & $23 . \ldots \ldots \ldots \ldots \ldots \ldots \ldots \ldots \ldots \ldots \ldots \ldots \ldots \ldots \ldots$ & 1 & 4 & 5 & 5 & 050 & 9125 . \\
\hline & 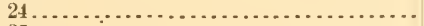 & 1 & $\ldots \ldots \ldots$ & 1 & 1 & 017 & 9126 \\
\hline & 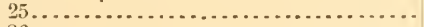 & 1 & ......... & 1 & 1 & 017 & 9124 \\
\hline & 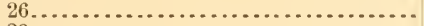 & 2 & & 7 & 7 & 012 & 9126 \\
\hline & 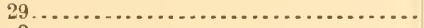 & 2 & 6 & 8 & 8 & 012 & 9126 \\
\hline & 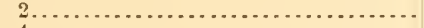 & ... & 2 & 2 & 2 & 100 & 9130 \\
\hline & 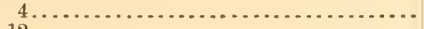 & 4 & .......... & 4 & 4 & 014 & 9136 \\
\hline & 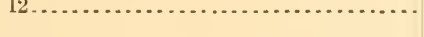 & 4 & & 6 & 6 & $\mathrm{~N}^{0} \mathrm{~N}^{10}$ & 9128 \\
\hline & 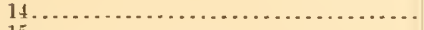 & 3 & 7 & 10 & 10 & 124 & 9149 \\
\hline & 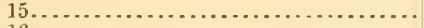 & 7 & 7 & 14 & 14 & 124 & 9149 \\
\hline & $16 \ldots \ldots \ldots \ldots$ & 1 & 3 & 4 & 4 & 124 & 9150 \\
\hline & $17 \ldots \ldots \ldots \ldots$ & 3 & 5 & 8 & 8 & 127 & 9149 \\
\hline & 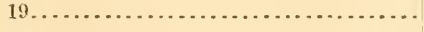 & 1 & .......... & 1 & 1 & 140 & 9202 \\
\hline & 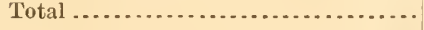 & 85 & 139 & 224 & 224 & 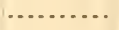 & ...... \\
\hline
\end{tabular}

District and Port of San Francisco.

I, IV. P. Noyes, master of the American sehooner Prosper, do solemuly and truly swear that the foregoing extract from the seal $\log$ book of the said vessel is correct, that the number and sex of seals taken on the days specified, with the latiturle and longitude, and all other particulars as herein expressed, are true to the best of my knowledge and belief, so help me God.

W. P. Noyes, Master Schooner Prosper.

Subscribed and sworn to before me this 15 th day of December, 1897.

N. S. FARLEY, Deputy Collector of Customs. 
Table C.-Log Extries of American Vessels Exgagei in Pelagic Sealing WRING THE SEASON OF 18:7, ETC.-Continuer.

Custom-House, San Francisco, December 16, $189 \%$.

I hereby certify that the foregoing is a true copy of the origiual abstract from the official seal $\log$ of the American schooner Prosper, and that it has been compared with said ofticial seal log and found to be correct.

I further certify that said official seal $\log$ is on file in this office.

I [SEAI.]

N. S. Farler, Deputy Collector of Customs.

Tessel landing her catch at Lnga, Alaska.

Name.

Males. Females. Total. Shot.

Rattler $a$

$\begin{array}{lll}6 & 187 \quad 193\end{array}$

193

a These skins were lanted and examined at the custom-house, Unga, Alaska, and subsequently shipped to San Francisco.

ARSTIRACT FROM THE SEAL LOG BOOK OF THE SCHOONER RATTLER, ENGAGED IN FIR-SEAL FISHING UNDER SPECIAL LICENSE ISSUED AT SAN FRANCISCO.

[Cleared from san Franciscu on Jan. 16,1897; arrived from sea on sept. 16,1897; entered at cnstomhouse, San Franeisco, on Sept. 17, 1897.

\begin{tabular}{|c|c|c|c|c|c|c|c|}
\hline & Date. & $\begin{array}{l}\text { Males } \\
\text { taken. }\end{array}$ & Females & Total. & Shot. & Latiturle. & Longitude. \\
\hline & 1897. & & & & & 1. & IV. \\
\hline Jan. & 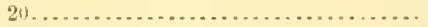 & $\ldots$ & 6 & 6 & 6 & 3736 & 12347 \\
\hline & 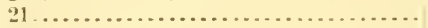 & $\ldots \ldots$ & 2 & 2 & 2 & 3657 & 12245 \\
\hline & 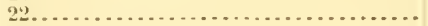 & $\ldots . . .$. & 6 & 6 & 6 & 3633 & 12226 \\
\hline & 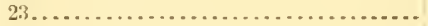 & $\ldots \ldots \ldots$ & 7 & 7 & 7 & 3645 & 12248 \\
\hline , & 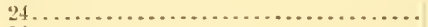 & ........ & 1 & 1 & 1 & 3628 & 12220 \\
\hline & 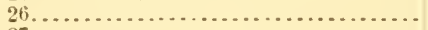 & 1 & 5 & 6 & 6 & 3713 & 12247 \\
\hline & 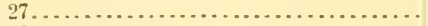 & $\ldots \ldots$. & 1 & 1 & 1 & 3755 & 12354 \\
\hline Feb. & 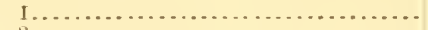 & ........ & 1 & 1 & 1 & 3806 & 12330 \\
\hline & 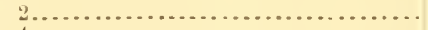 & ........ & 5 & 5 & 5 & 3733 & 12323 \\
\hline & 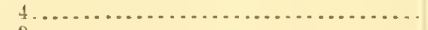 & ........ & 1 & 1 & 1 & 3805 & $123+2$ \\
\hline & 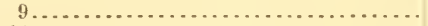 & $\ldots \ldots$. & 6 & 6 & 6 & 3624 & 12214 \\
\hline & 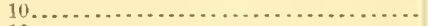 & $\ldots \ldots \ldots$ & 10 & 10 & 10 & 3519 & 12135 \\
\hline & 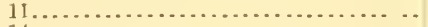 & $\ldots \ldots \ldots$ & 1 & I & 1 & 3522 & 12045 \\
\hline & 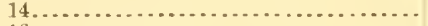 & $\ldots \ldots$. & 1 & 1 & 1 & 3512 & I20 38 \\
\hline & 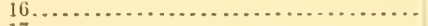 & ........ & 3 & 3 & 3 & 3525 & 12215 \\
\hline & 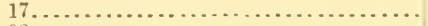 & $\ldots \ldots \ldots$ & 9 & 9 & 9 & 3612 & 12232 \\
\hline & 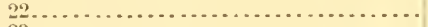 & ........ & 11 & 11 & 11 & 3732 & 12345 \\
\hline & $23 \ldots \ldots \ldots \ldots \ldots \ldots \ldots \ldots \ldots \ldots \ldots \ldots \ldots \ldots$ & 1 & 7 & 8 & 8 & 3740 & 12346 \\
\hline & 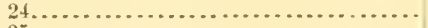 & 1 & 32 & 33 & 33 & 3753 & 12320 \\
\hline & 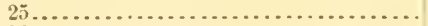 & 1 & 6 & 7 & 7 & 3801 & 12340 \\
\hline & 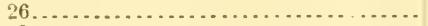 & $\ldots \ldots \ldots$ & 3 & 2 & 2 & 3812 & 12332 \\
\hline MIar. & 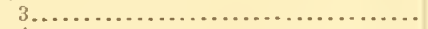 & $\ldots \ldots \ldots$ & 2 & 2 & 2 & 4051 & 12446 \\
\hline & 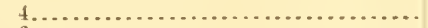 & $\ldots \ldots \ldots$ & 6 & 6 & 6 & 4107 & $12 \div 14$ \\
\hline & $6 \ldots \ldots \ldots \ldots \ldots \ldots \ldots \ldots \ldots \ldots \ldots$ & $\ldots \ldots \ldots$ & 3 & 3 & 3 & 4148 & 12448 \\
\hline & 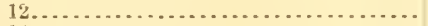 & ........ & 29 & 29 & 29 & $43 \quad 39$ & 12431 \\
\hline & 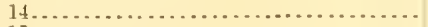 & 1 & 6 & 7 & 7 & 4336 & 12435 \\
\hline & 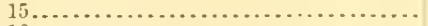 & $\ldots \ldots \ldots$ & 9 & 9 & 9 & 4335 & 12440 \\
\hline & $16 \ldots \ldots \ldots \ldots \ldots \ldots$ & 1 & 5 & 6 & 6 & 4336 & 12440 \\
\hline & $21 \ldots \ldots \ldots \ldots \ldots \ldots \ldots \ldots \ldots \ldots \ldots$ & & 4 & 4 & 4 & 4434 & 12438 \\
\hline & Total ........ & 6 & 187 & 193 & 193 & & \\
\hline
\end{tabular}

District and Port of San liraxcisco.

1, Fed Neilson, master of the American schooner Ratllo, do solemnly and truly swear thit the foregoing abstract from the seal $\log$ look of the said vessel is correct; that the number and sex of seals taken on the days specifier, with the latitude and longitude, and all other particulars as heroin expressed, are true, to the best of $\mathrm{my}$ knowledge aud belief, so help me God.

Subscriber and sworn to before me, September $17,1897$.

Fired. NeILson, Master.

N. S. Farler, Deputy Collector.

Custom-House, SAx Fraxcisco, September 18, $189 \%$.

I hereby certify that the foregoing is a true extract from tho official seal $\log$ book of the Amorican schooner Rattler, and that said official log book was filed in this office, Scptem ber $17,1897$.

N. S. Farder, Deputy Collector of Customs. 


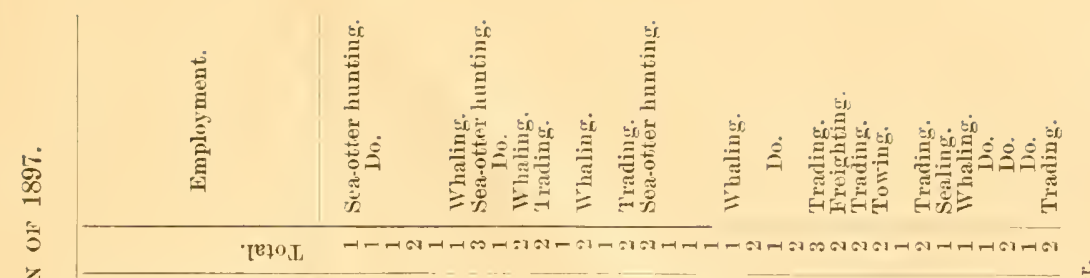

.

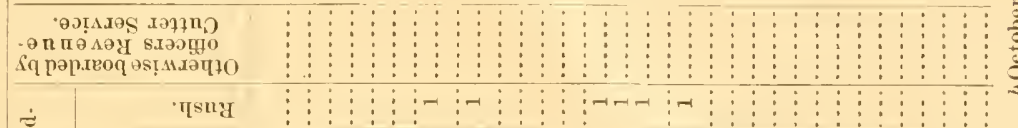

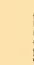

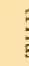

.

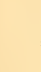

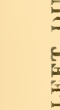

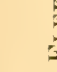

을
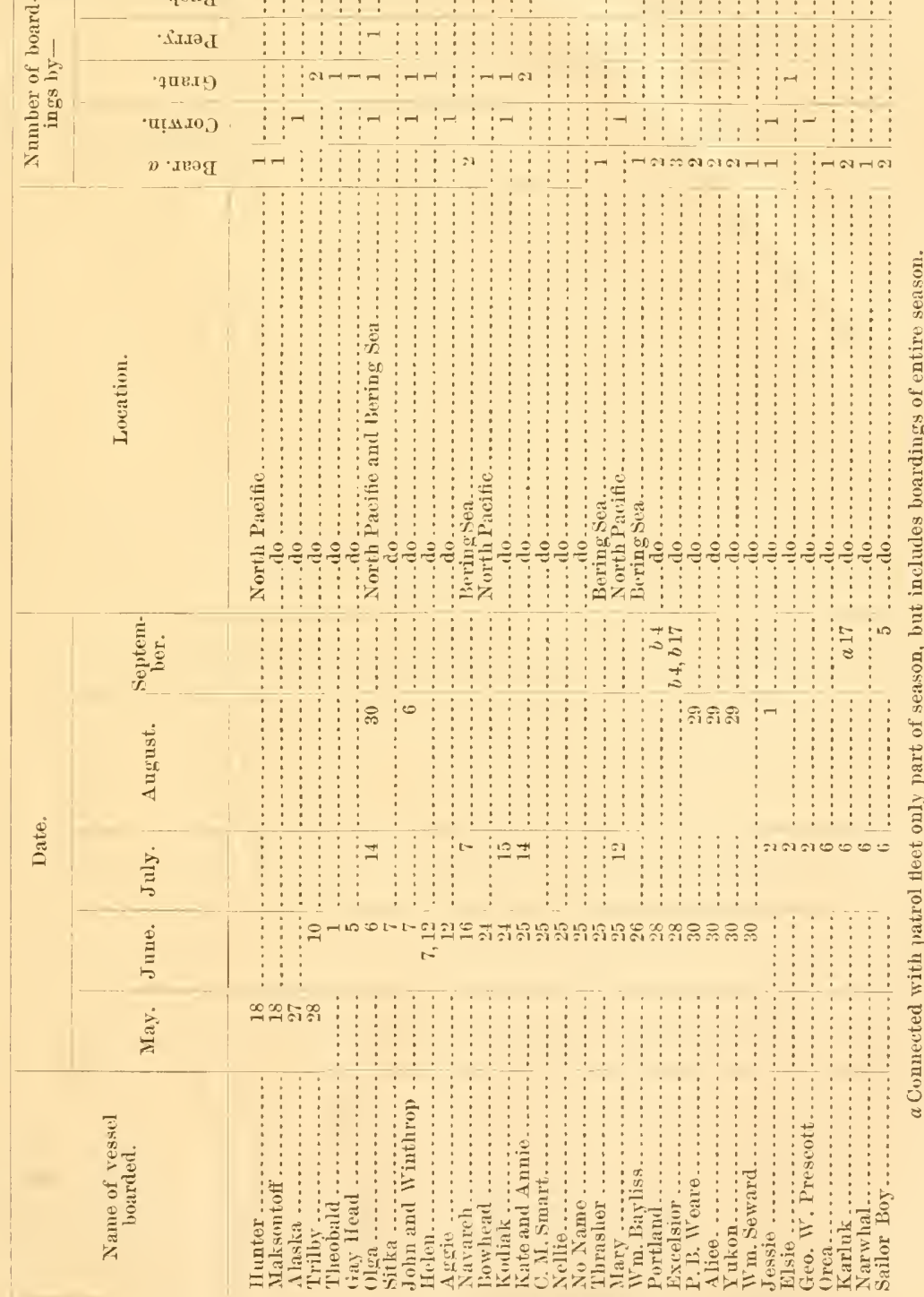

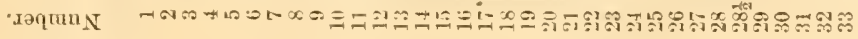




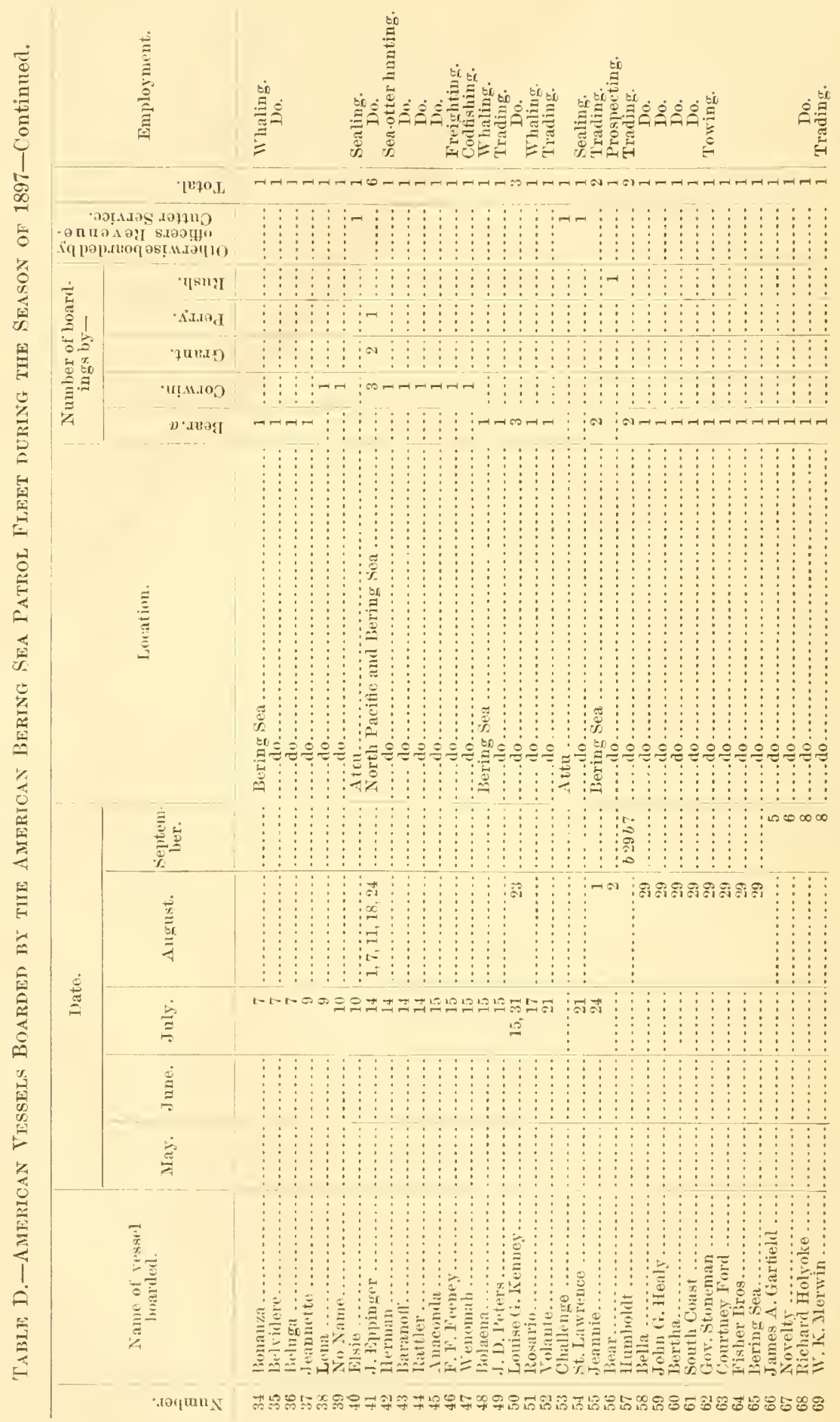



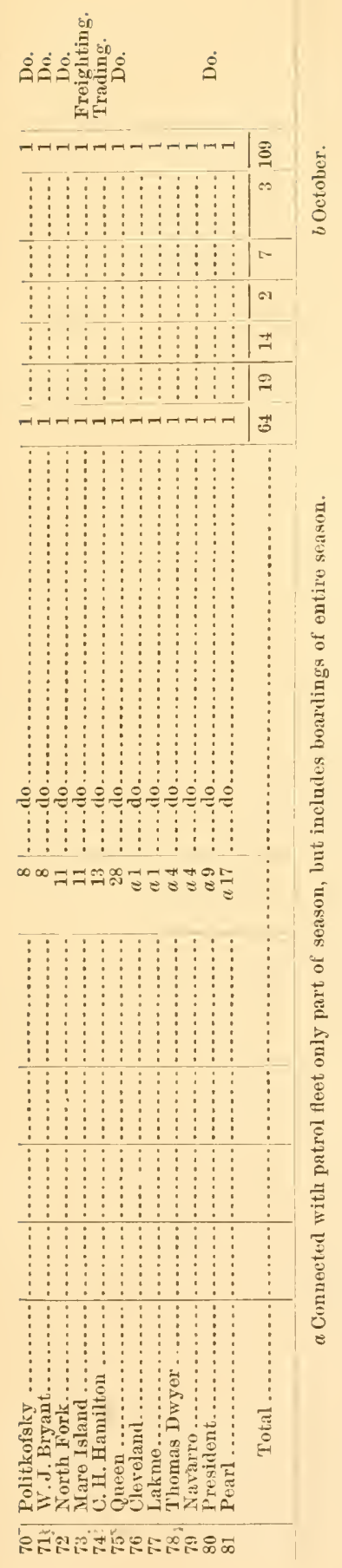


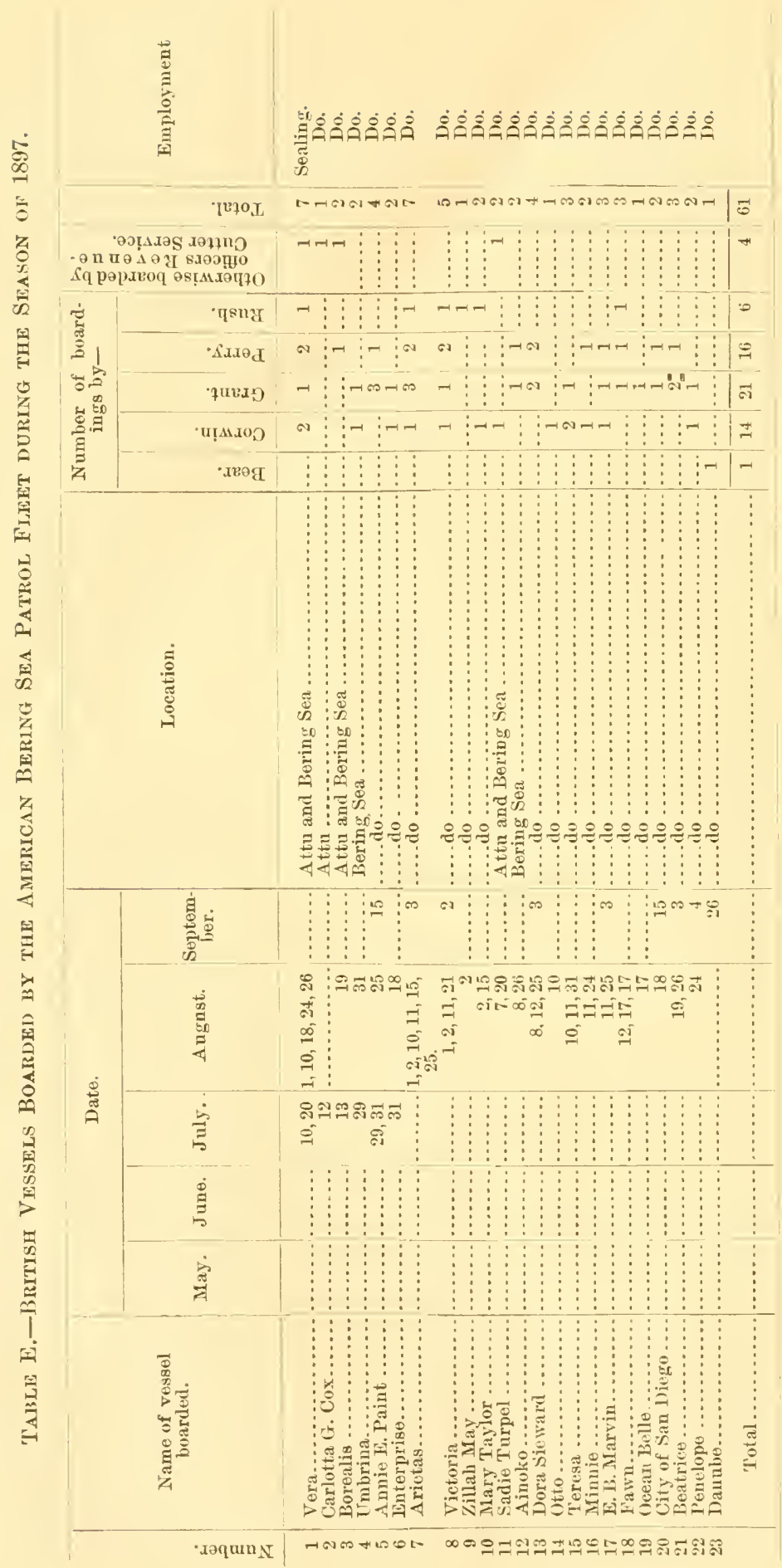




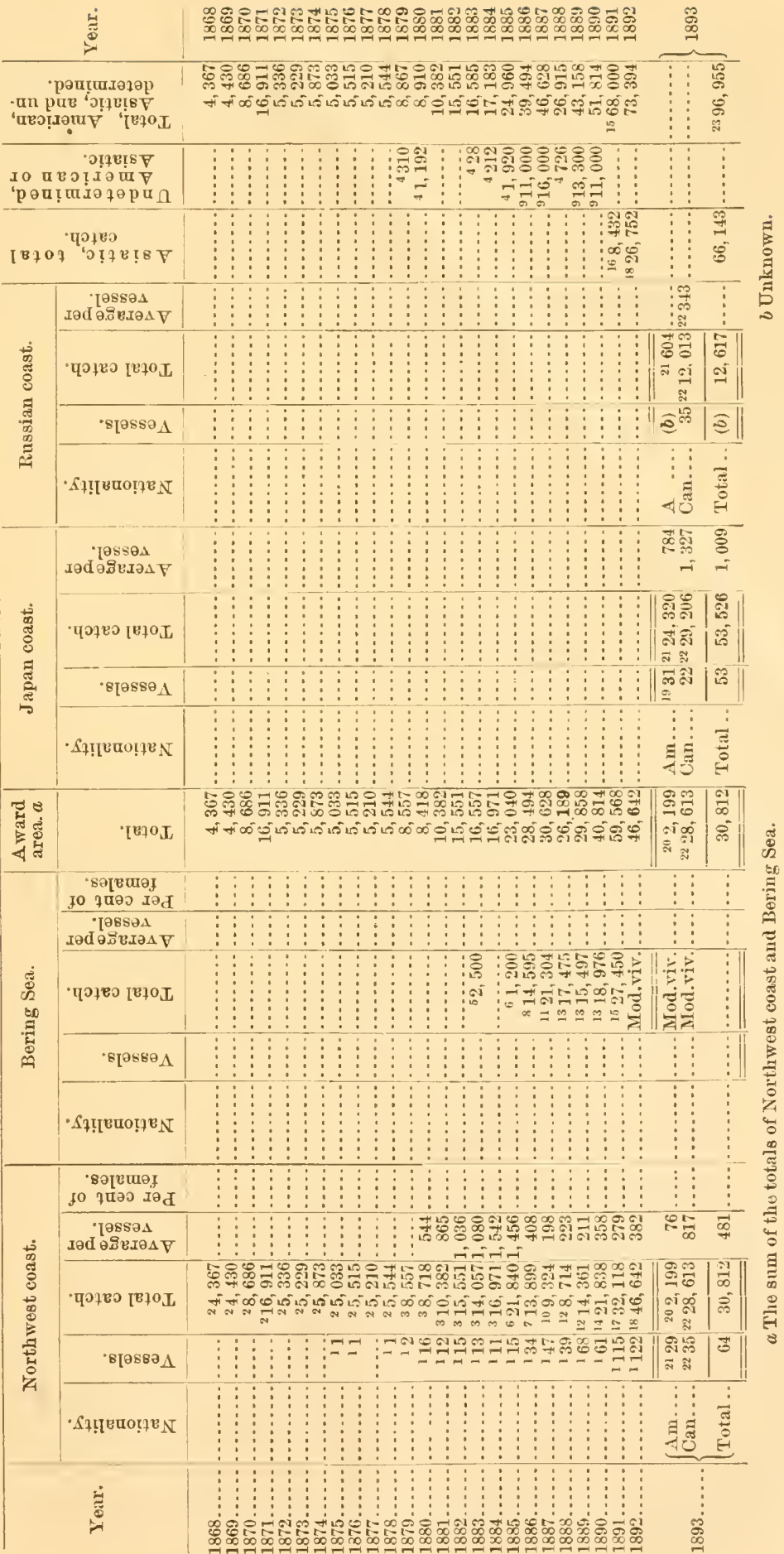




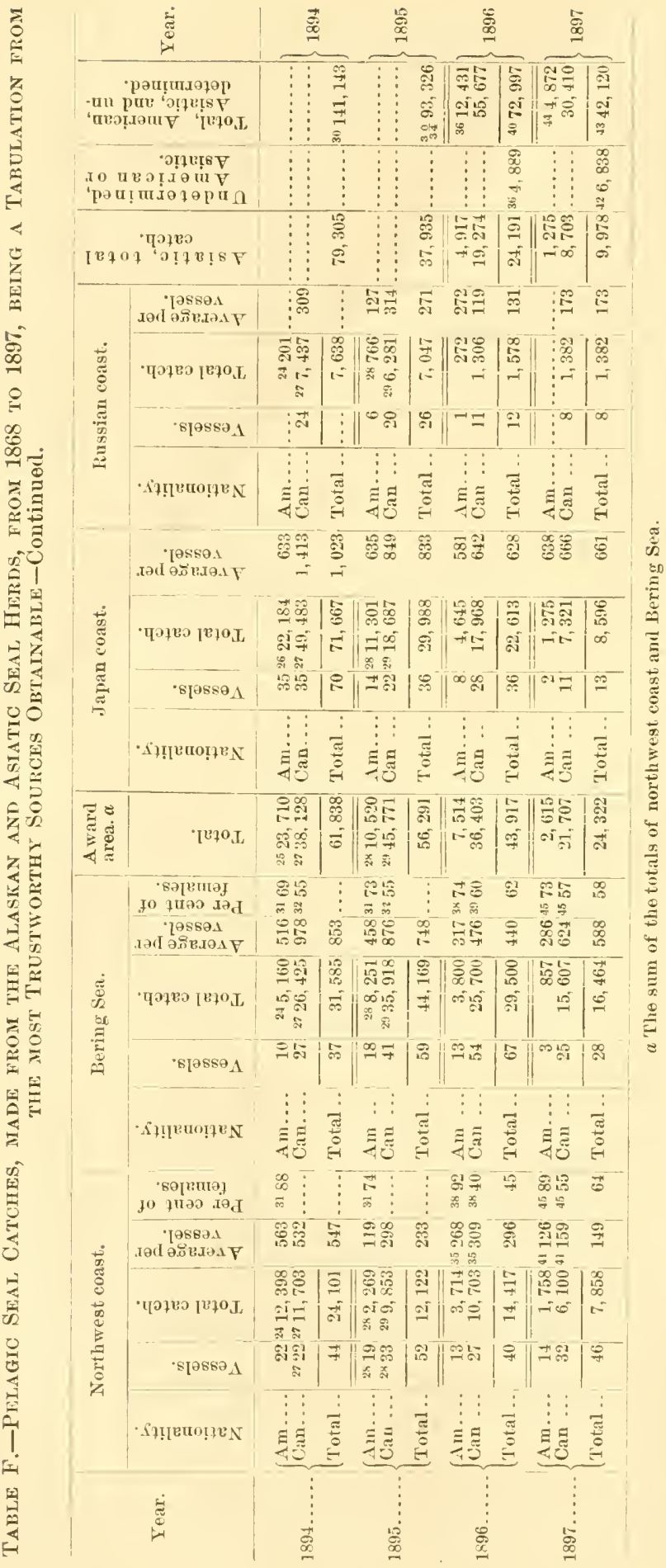




\section{Table G.-Authoritis fol Figures Usen in Tables B and F.}

[In ruoting from Fur.Seal A rbitration Pajers, the first edition is always referred to.]

1 Fur-seal Arbitration. A p. to U.S. Case, vol. 1, p. 591.

2 Catches for rears 1898 to 1879 , inchusive, are made uj, of Northwest coast catehes (Fur-Seal Arbitration: British Commissioners' Rejort, p. 207 et seq.) ; Indian eanoe eatehes (British Commjssioners' Report, pp. 207;208), and skins obtained through the Hudson Bay Company's trading stations (British Commissioners' Report, p. 213).

${ }^{3}$ Catches of pelagic sealers and Indian canoes (British Commissioners' Report, pp. 207, 208), and returns from Hudson Bay Company's posts (British Commissioners' leport, p. 213).

"San Franciseo custom-house records; Deputy Collector Jerome's letters of February 26 et ser., 1892 on file in Treasurs Department.

${ }_{5}^{5}$ Catch of schooner City of San Diego (British Commissioners' Report, p. 208).

${ }_{6}$ Catches of pelagie sealers in North I'acitic and Bering Sea (British Commissioners' Rejort, p. 209), and returns from Hudson Bav Company's bosts (British Commissioners' henort, 1. 2]3).

'Catches in North Pacific from all sourees (British Commissioners' Report, pp. 210, 213).

* Marketerl catches from Bering Sea (British Commissioners' Report, p. 210) plus 2,000 skins seized on schooners Onvard, Thornton, Tarolena, ant san Diego (H. H. If Intyre's manuseript report to Alaska Commercial Company, a copy of which is in possession of lepartinent).

9 Sritish commissioners' estimater cateh of American ressels in all loealitieg (British Commissioners Report, p. 212).

10 North Pacific catches (British Commissioners lieport, 1P. 210, 213).

1 J Jarketed catches from liering Se: (l'ritish ('ommissioners' I'́purt, p). 210) plus 8,910 skins seized in Bering sea and nuccounted for by British commissioners; 11,901 skins were seized that year (United States Counter Case, p.337), and the British commissioners, on bage 210 of their report, account for 2,991 of them.

${ }_{12}$ North Pacific catehes (British Commissioners' lieport, j]. 2211, 213).

13 Bering sea catches (British Commissioners' Tieport, pp. 211,212 ).

${ }^{4}$ North Pacihe catches (hritish Commissioners' licport. pl), 211, 213).

$15^{\prime}$ This fignre, 27,450 , is the sum of the tigures 22,530 and 4,920 , the origin of which will be found under note 16. The British commissione 5 s, on plage 18 of their report, gire the approximate total cateh as 68,000 .

${ }_{16}$ In a letter from the British foreign office to the secretary of State, dated Mas 17, 1895, the Bering Sea catch of British vessels for $189 \mathrm{H}$ is nuoted at $29.14 \mathrm{j}$. It has been fornd by this Department that these figures represent the total catch in Bering sea-that is, including seals killed off the western side, in Russian waters, as well as oin the eastern side, which afterwarls became the award area. This is borne out by the fict that it appuars by the British case before the tribmual at Paris that 41 vessels were warned out of the American sille of Beriug Sea between June 29 and August 15, 1891, under the modus vivendi of . lum 15 of that year. It is certain that many of these resselscrossed over to the Russian side of Bering Sea and rontinned sealing nntil the rlose of the season.

Statistics made by Mr. Alfred Fraser, now in Jossession of the Treasury lepartment, show that 8,432 skins were thus taken on the wentern sicle of Bering sea in litssian waters. Of these, 6,616 were taken by British vessels and 1.816 by American vessels. We should, therefore, dednct from the British figures $(29,146)$ the sum of 6,616 , leaving 22,530 as the British eatch in the award area-that is, the eastern side or Bering Sea- ior the year 1891 .

It further appears from $M 1 \mathrm{r}$. Fraser's figures that the Anerican catch in Bering sea in 1891 was 6.736, of which 1816 were taken in Russian waters and $4,9 \div 0$ in the award area Adding to the corrected British catch, 22.530, the catch of the Auerican ressels, 4,920, we have 27,450 -as the total catch of British and American vessels in that part of lbering sea known as the award area for the year 1891.

In the report of the Committe on Ways ant Means to accompany H. R. 8909, Fifty-third Congress, thirt session. Report Fo. 184!3, the eatch in Bering sea for the year 1891 was given as 23,041, on the anthority of the 'Treasury Department. These figures inchided only the returns of Litish vessels, as no reliable returus as to Imeriean ressels were then in the possession of the Department. 'The result was reached by dedurting from the estinnte giren by Consul NJejers in his report (Inited States counter case), 28,605. a number of skins ustimated to har heen taken of the liussian coast. 'This estimate was reached by a careful examination of all catches referred to in the aftidavits and other papers in the case and connter ease of the Tnited states and Great Britain, exclnding those which were ciaimed to hace heen taken ofl the liussian coast.

That the British returns (above citert), 29,146 , inchude seals taken on the western side of Bering Sea, from the liussian berd, will appear, as above statcd, Jrom the fact of the warning of said vessels, under the modus vivendi, and their subsianent erosing to the Iinssian coast.

'The report of the minister of marine and fislieries of Camada for 1891 eredits none of the catch to Russian waters. In 1892 , bowever, said report credits 14,815 skins ont of a total of 53,912 from said A siatie shores. The fact that this large eateh was marle in 1892 joints strongly to similar catches in the year 1891, which are enfirmed loy the ahore-mentioned evidence.

17 Obtained by subtracting the total of 27.450 and 8,432 from 68,000 .

18 see Uniterl states connter ense, page 408.

is Taken from Alfred Fraser's estinates lor A merican sealing fleet in Asiaticwaters. Skins entered in Linited states ports.

20 T'lie smatlness of the number 2,199 stiggests that either many of the ressels after clearing sailed directly fur the Japan coast, or else the catches off the Norlhwest coast were transshipped at Japan ports.

${ }^{21}$ The American cateh for 1893 is based npon statistics compiled hy $\mathrm{A}$. Fraser and on file in the Treas ury Department. The United States consul at Vietoria states (Consular Reports No. 161, p. 279) that American scheoners in 1893 transshipped at Yokohama and Hakodate between 17,000 and 18,000 skins. This is turther contirued by the reprort of the ('anadian department of marine and fisheries for 1893 , page clxviii, which gives the ratch of American ressels landed at Hakodate as 18,587 .

${ }_{22}$ The figures tor the catches of Canadian ressels are taken from the report of the Canadian depart ment of marine and fisheries for 1893, pitge elxrii.

${ }^{23}$ 'The London trade sales for 1893 aceount for the disposition of 109,669 pelagie skins.

24 Compiled from the reports of collectors at ports of entry on the Pacific coast. These reports are on file in the Treasury Department.

${ }_{25}$ The tigure $2: 310$ is ohtained by taking the 6.436 skins noted nuder the caption "Locality undeter minerl "in the letter of the Seeretary of the Treasury to Congress dated Jannary 21, 1895 (Fifty third Concress, third session. Ex. Doc. 243), and dividing them between the A siatic and American herds in similar proportions as the other skins landed at United States ports in United states sealing vessels during 1894. The result would be: Imerican herd, 6,152; Asiatic, 684. Adeling 6,152 to the catch on 
the Northwest coast $(12,398)$ alrealy given and the liering Sea catch $(5,160)$ already given, we have the total 23,710 .

${ }_{26}$ Made up of skins as per records of collectors of enstoms on the Pacific Coast, which credit 1,500 to Asiatic waters; 684 skins, previonsly referrerl to in note 25 , and the 20,000 skins which it is cstimated were transshipped in Japan (Ex. Doc. 243, Fitty-thirl Congress, third session. "Notes con. cerning catch for $1894, "$ p.4).

27 Taken from report of Canadian department of marine ancl fisheries for 1894 , page 9.

The figures 26,425 inclnde one Americun vessel, whose catch was 81 skins.

The figures 49,813 contain the catches of three American ressols, which aggregated 490 skins.

The facts in the two foregoing paragraphs are given in a report of Fisheries Commissioner Costigan to the Governor-General of Canada, under date of January 9, 1895, lage 9.

${ }_{28}$ Reperts of collectors of enstoms at American ports of entry on the Pacific Coast.

${ }_{29}^{2}$ Ofticial statement sent by Lnited States Consul Roberts at Victoria, under date of November 15 , 1895. and on file in the Treasury Department.

30 Desul 1 ups. - The grand totals for 1894 and 1895 do not include dead pups. In 1894 , by careful estimate based upon partial count, 20,000 pups perisherl, and in 1895, by actual count, 28,000. This would swell the known deaths, exclusive of the lant eatch, in 1894 to 161,143 and in 1895 to 121,326 . see note 37 for slead pups for 1896 .

The pelagie catch for 1895 is further increased by a catch of about 10,000 skins taken by ressels clearing from Japanese ports.

31 From returns of Tnited States inspectors who examinerl skins landed in United States ports.

32 From official returns of collector of eustoms, Victoria, Britısh Columbia. Sikins not inspected.

${ }^{33}$ Total sealing ressels in awarl area.

\begin{tabular}{|c|c|c|c|c|c|}
\hline & 1893. & 1894. & 1895. & 1896. & 1897. \\
\hline American....... & 29 & 30 & 32 & 18 & 16 \\
\hline Canadian ....... & 35 & 32 & 49 & 59 & 39 \\
\hline Total & 64 & 62 & 81 & 77 & 55 \\
\hline
\end{tabular}

Total sealing ressels in isiatic waters.

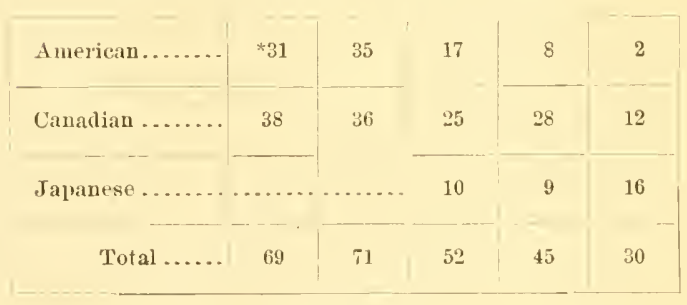

* Estimated.

Total seuling ressels in Asiatic and Imerican waters.

\begin{tabular}{|c|c|c|c|c|c|}
\hline American....... & 28 & 35 & 35 & 21 & 16 \\
\hline Canadian ....... & 56 & 60 & 62 & 66 & 41 \\
\hline Japanese.... & & & 10 & 9 & 16 \\
\hline Total ..... & 84 & 95 & 107 & 96 & 73 \\
\hline
\end{tabular}

${ }^{34}$ Mr. Alfred Fraser estimates from trade returns that the pelagic ratehes from the Anerican and A siatic lierds aggregated 98,204 skins in 1895 . 
COMPARISON OE CATCHES OF PELAGIC SEALERS IN 1894, 1895, 1896, AND 1897.

1891.

NORTHWEST COAST.

Forty-four vessels took 24,101 seals, being an arerage of 547 skins per vessel.

BEIRING SEA.

Thirty-seven ressels took 31,585 seals, being an arerage of 853 skins per ressel.

1895.

NORTHWEST COANT.

Fift 5 -two ressels took 12,122 seals, being an arerage of 233 skins per vessel, or a decrease of 57 per cent as compared with 1894 .

BERING SEA.

Fifty-nine ressels took 44,169 seals, being an average of 748 skins per ressel, or a decrease of 12 per cent as compared with 1894 .

1596.

NORTHWEST COAST.

Thirty-nine ressels took 11,837 seals, being an arerage of 304 skins per ressel, an increase of 30 per cent as compared with 1895 .

BERING SEA

Sixty-seren ressels took 29,500 seals, being an arerage of 440 skins per vessel, or a decrease of 41 per cent as compared with 1895 .

\section{7.}

NORTHWEST COAST.

Forty-six vessels took $6,840 \mathrm{skins}$, an average of 149 skins per vessel, or a decrease of 51 per cent as compared with 1896.

BERING SEA.

Twenty-eight vessels took 16,464 skins, an average of 588 skins per ressel, or an increase of 34 per cent as compared with 1896 .

PERCENTAGE OF FEMALES IN PELAGIC CATCHES IN 1894, 1895, 1896, AND 1897. NORTHWEST COAST.

$18 ! 1$.

American vessels, 88 per eent. British give no figures. See notes 31 and 32.

\section{5.}

American ressels, 74 per cent. British give no figures. See notes 31 and 32.

1896.

American ressels, 93 per cent. British ressels, 40 per cent. See notes 31 and 32.

$18 ! 7$.

American vessels, 89 per cent. British ressels, 55 per cent. See notes 31 and 32.

BERING SEA.

1891.

A merican ressels, 69 per cent. British vessels, 55 per eent. See notes 31 and 32.

1595.

American vessels, 73 per cent. British ressels, 55 per cent. See notes 31 and 32.

$15 ! 6 \%$

American ressels, 75 per cent. British ressels, 61 per cent. See notes 31 and 32.

\section{7.}

American ressels, 73 per cent. British ressels, 57 per cent. Sce notes 31 and 32. 


\section{Explanatory notes relatiny to catch for 1536 .}

${ }^{35}$ In arerages per ressel relating to Northwest eoast eatch, the canoe catches are not included. British Colmmbia eanoe catch, 2,353 , included in Canadian Northwest coast total.

${ }^{36}$ Total catch of American and Canadian ressels for 1896 further inereilsed by a catch of 3,392 skins taken bs ressels clearing from. Tapanese ports, and of 1,497 skius taken by natives in the passes of the
Aleutian Islands. si The grrand tots

21,228 dead and to 1,546 dying at time of count the loss of pups on the I'ribilof Islands, amonnting to $3 \times$ tll log a to 1,546 dying at time of count.

3* All log entries relating to American pelagic eatch sworn to by masters of ressels, but most of 39 Proportion of females in all Canales upou examination of eatches by inspectors of 'seal skins,

Catches not officially inspected as to aex.

to Dita coucering inspected as to sex.

${ }^{40}$ Data concerning catches of American ressels in all waters for 1896 are based on rejorts from United States custom-houses, supplemented by intimmation collected by Mr. C. H. Townsend; data concerncatehes in 1895 of resels catces in 1895 of ressels belonging to Japanese purts, furnished by United States consular officers in Japsn. Catches of similar vessels in 1896 are from nnoffieial sources, are incomplete, and less than
number actually taken.

\section{Explanatory notes relating to catch for 189 .}

41 In a rerages per ressel relating to the northwest coast catch, the British Columbian canoe cateh, is not included.

${ }_{42}$ The total cath of the British Columbian and American fleets for 1897 is inereased further by the. catch of the Japanese sealiug fleet during the rear -16 vessels, 6,838 skins.

43 The data from which were compiled the statisties relating to the American cateh for 1897 were obtained from the official reports from T.S. custom-houses; the statistics of the British Columbian catch for the same period were obtained by the IT. S. consul at Tictoria, B. C., from custom-house records at that port; the figures showing the cateh of the Japanese fleet were furnished by Dr. Leon-
hard stejneger.

H4 The grand total of seals taken by United States vessels include 764 skins taken south of the award area by the schooners $J$. Eppinger and Lovisa 1), but undoulstedly from the herd frequenting the

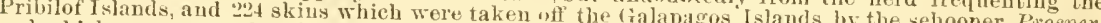
and whieh are supposed to be a species (arctocephiclus fhipas Pribilot Islands.

${ }^{45}$ Statements by masters of American vessels as to the sex of seals taken verified in every instance by examination by inspector's at ports of entry. Proportion of temales in Canadian catches taken from tigures submitted by U.S. consul (see note 43); eatehes not officially inspected.

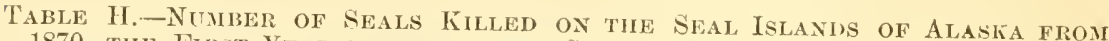
1870, THE FIRST YEAR 1,UIING WHICII SEALS WERE KILLED BY LESSEES DULY

LICLNSEI) BY THE GOYERNMENT, To 1897, BOTH INCLUSIVE, $a$

UNDER LEASE DATED AUGUST 3. 1870, TO THE ALASKA COMMERCTAL COMPANY.

\begin{tabular}{|c|c|c|c|}
\hline Year. & Seals taken. & Tear. & Seals taken. \\
\hline 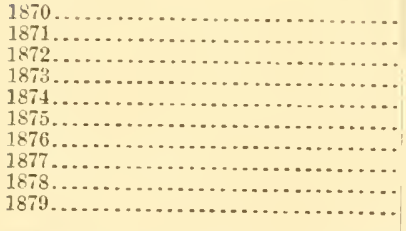 & $\begin{array}{r}23,773 \\
102,960 \\
108,819 \\
109,177 \\
110,585 \\
106,460 \\
94,657 \\
81,310 \\
109,323 \\
110,511\end{array}$ & 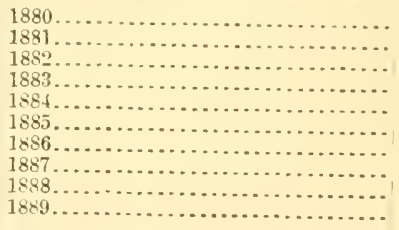 & $\begin{array}{r}105,718 \\
105,063 \\
99,812 \\
79,509 \\
105,434 \\
105,024 \\
104,521 \\
105,760 \\
103,304 \\
102,617\end{array}$ \\
\hline
\end{tabular}

( Data as to the killings during 1870-1889, both inclusive, compiled from othicial records on st. Paul and St. George islands, published in Reprint of Proceedings of 'Tribunal of Arbitration at Paris, vol. UNDER LEASE DATED MARCH 12, 1890, TO TIE NORTII AMERICAN COMMERCIAL

Year. Seals taken.

Year. Seals taken.

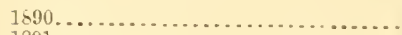

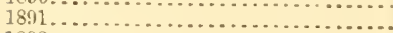

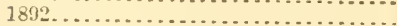

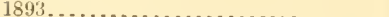

\begin{tabular}{|c|c|}
\hline 20,995 & 1894. \\
\hline 13,482 & 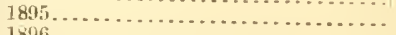 \\
\hline $\begin{array}{l}\tau, 549 \\
\tau, 5110\end{array}$ & 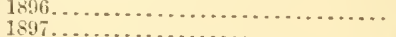 \\
\hline
\end{tabular}

a The figures her' given for 1890 and subsequent years inchade only those seals the skins of which were aceepted as a part of the annul quotas of the North Ameriean Commercial Company. 

$M B-38$. 





\section{0}

2.

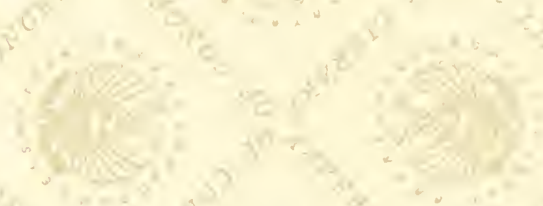

wions

(1)

$+4$

3
$\rightarrow \quad 3$
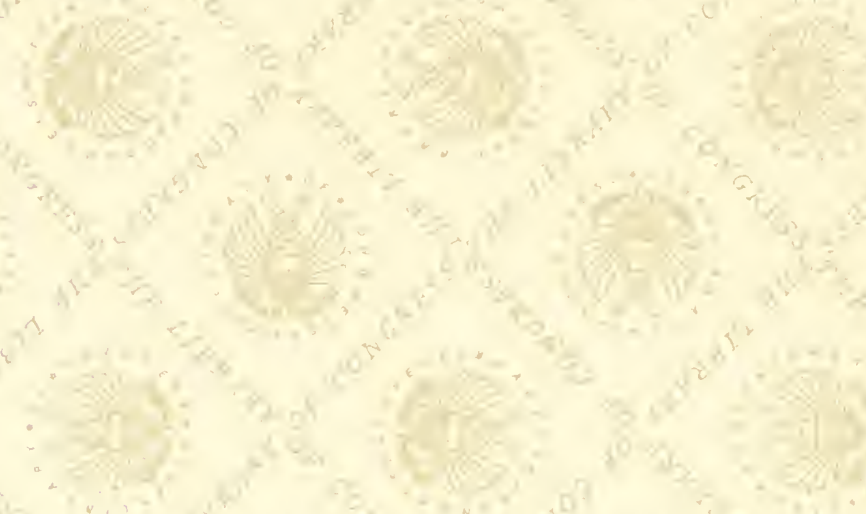

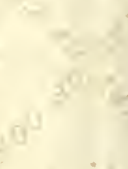

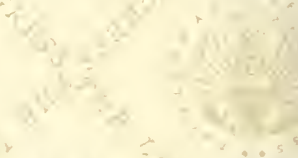

- 3

$x_{2}^{2}$

$=16+1$

Yin-

$10 \times 1=$

$3^{2}$
8
8
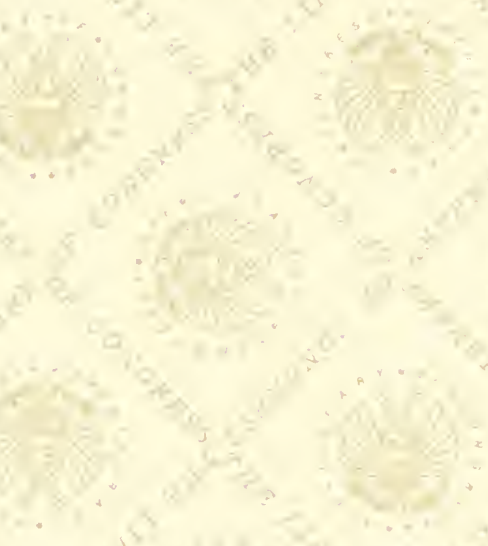

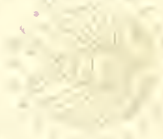

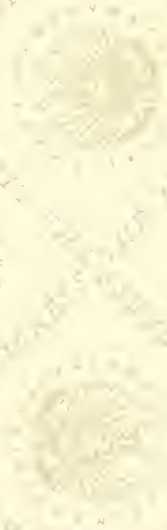

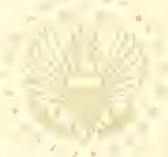

$6^{5}-13$
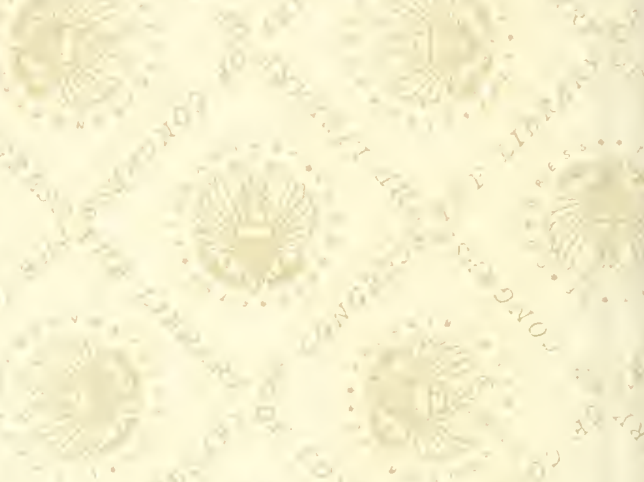

$x^{2}+y^{2}=$
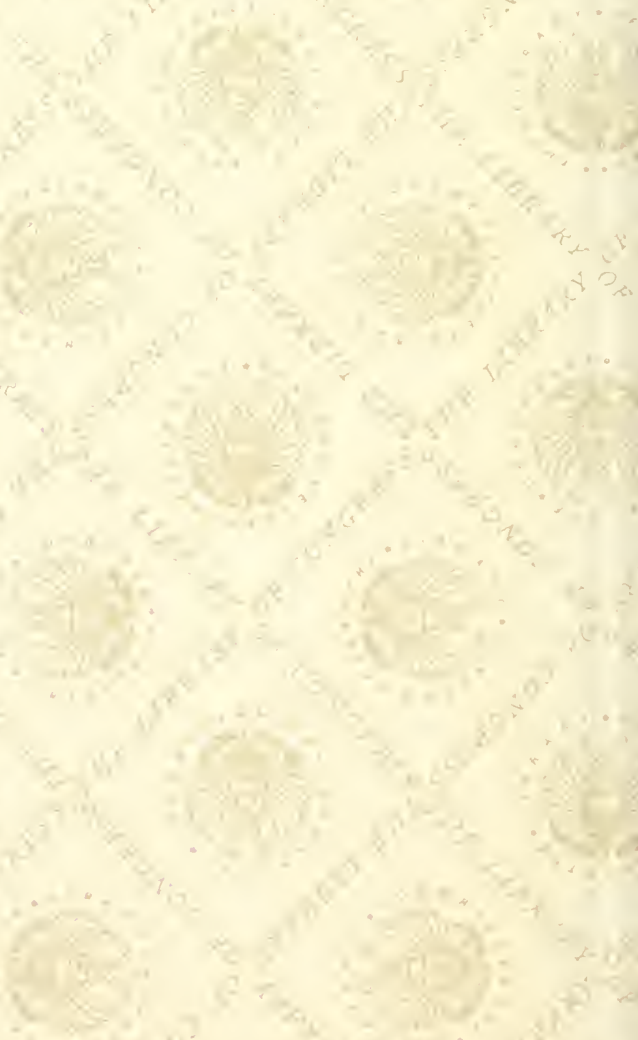

$\frac{1}{4}$
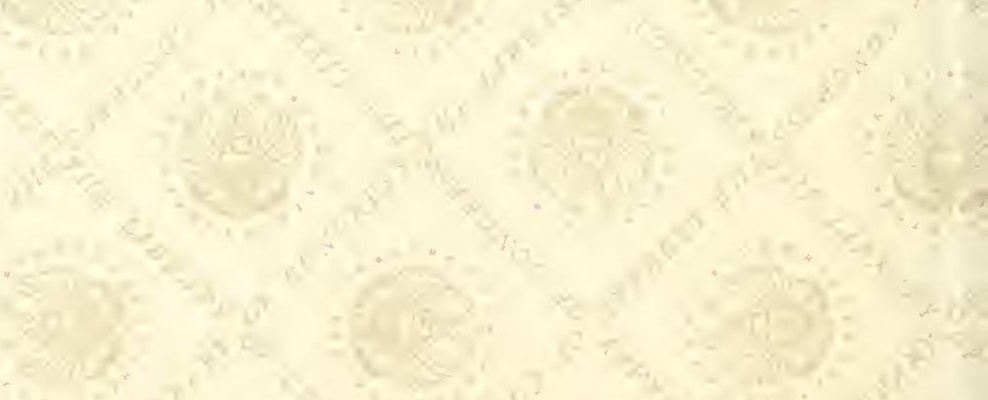

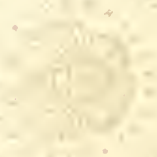





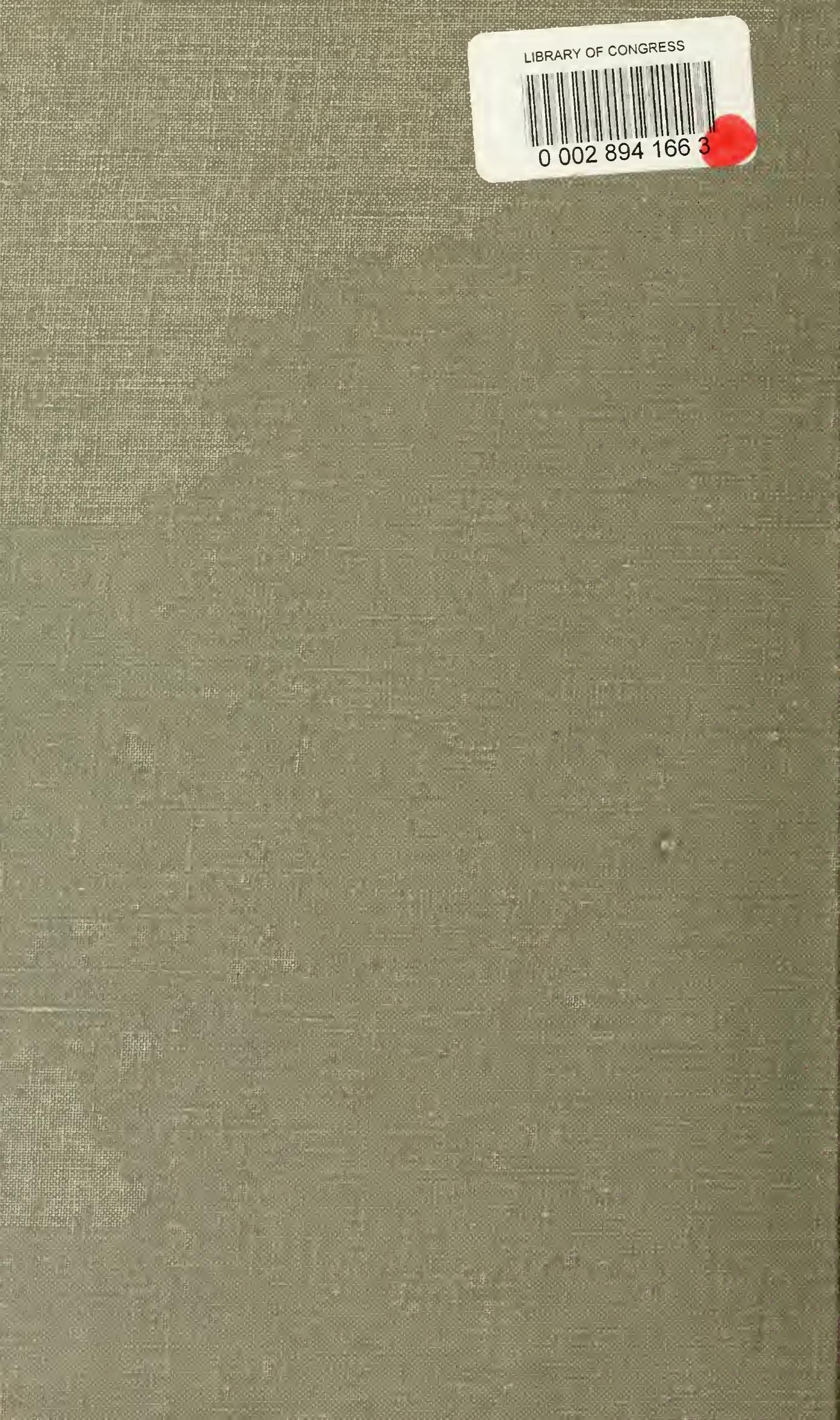

